DOI https://doi.org/10.30898/1684-1719.2021.3.8

UDC 621.396

\title{
COMPARATIVE ANALYSIS OF CYLINDRICAL AND PLANAR AESA IN 3D SUVEILLANCE RADAR
}

\author{
D. Paunović \\ MITEC doo, Branka Miljkovica 27, 11060, Beograd (Palilula) Serbia
}

The paper was received on January 26 2021, after correction - on February 9, 2021

\begin{abstract}
A modern rotating 3D surveillance radars scan azimuth by mechanical rotation, and scan elevation using Active Electronic Scanning Array (AESA) in Multi Beam Receive Mode (MBM). Radars with fixed cylindrical AESA and four-sided prismatic antenna, with 4 flat AESA, scan azimuth electronically, without mechanical rotation. The most significant advantage of electronic scanning is the possibility of Multi-mode operation: surveillance targets in the far zone and targeting targets in the near zone. However, electronic scanning also brings problems. A main beam of planar AESA spreads and lateral lobes increase when the radiating direction increases. An original arrangement of shifted array to reduce lateral lobes has been proposed. The cylindrical array has a constant shape of pattern during azimuth scanning. But, for both prismatic and cylindrical AESA, the beam deforms during scanning in vertical plane, so limits the elevation scan. Also, the complexity and price of fixed AESA is significantly higher compared to the rotating one. In order to enable the selection of the optimal solution for a specific application, the comparative analysis of advantages and disadvantages for cylindrical, prismatic and rotating AESA is done. The original configuration of the cylindrical AESA for Very Fast Scanning in Near-zone has been proposed.
\end{abstract}

Key words: cylindrical array, scan speed, beam deformation, surveillance time, multi-mode, multiple beam forming, active electronically scanned array (AESA). 


\section{Introduction}

Most modern medium-range 3D radars, up to $500 \mathrm{~km}$, are based on a flat array that rotates around a vertical axis, like AN/TPS-80. Electronic scanning is performed only in elevation. Mechanical rotation in the azimuth leads to wear and increased energy consumption, especially for large radar structures at low frequencies. The speed of rotation in the horizontal plane is continuous and uniform. The radar must rotate fast to be able to track close targets which quickly changes position's angle. This, in turn, decreases a range.

Electronic scanning radars have the ability to scan in discrete azimuth directions. There is a possibility of skipping a sector that is not of interest. By changing the mode of operation by pulse to pulse, a multi-mode is possible: surveillance targets in the far zone and targeting targets in the near zone.

A comparative analysis of mechanically rotating and fixed AESA [1] justifies the introduction of radar with electronical scanning. However, so far only a few AESA-based surveillance radars have been published, such as:

- Raytheon Company's 360-degree for the German Air Force,

- APAR shipborne multifunction 3D radar by Thales,

- AN/SPY-1 navy 3D radar by Lockheed Martin,

- AN/FPS-132 early warning radar,

- SMART-L by Thales,

- ELM-2138M from Israel,

- Osprey, and Seaspray 7500E V2 by Leonardo,

- Chinese four S-band Type 346B AESA for 055 destroyer.

So far, no long-range surveillance radar with cylindrical AESA has been published. Why is that so? Are the reasons limitations due to beam deformation or complexity of realization?

Theoretical analysis for flat and cylindrical AESA has long been available [2], and there are many papers for synthesis [3-5]. Some problems with AESA have been hinted at, such as a decrease in gain and an increase in lateral lobes. In order to see 
the problems in the implementation of AESA, a qualitative and quantitative analysis is needed.

On the contrary to the general analysis [1], a program for the analysis of individual cylindrical and flat AESAs has been developed. The program calculates the range, surveillance time, gain, lobe level, and shows the distortion of the beam. Based on these results, the limitations in the application can be seen. Since the pattern is the result of the influence of a large number of elements, it is very difficult to predict the limits of AESA application without a pattern calculation program.

Since it was shown in [1] that in terms of surveillance time there is no significant difference whether the cylindrical AESA works with 2, 3 or 4 beams, we will observe only the array with 2 beams. It has also been shown that a four-sided prismatic AESA is better than a three-sided one. Therefore, the four-sided prismatic AESA was analysed. Two variants of the prismatic configuration are observed: the one with same size as the cylindrical one and other with the same number of TR modules. In terms of pattern analysis, radar with a rotating flat AESA can be considered as a variant of the fixed flat AESA at azimuth of $0^{\circ}$.

It is assumed that each radiating element in AESA has its own TR module.

\section{Construction of AESA}

Prismatic AESA for $360^{\circ}$ scanning surveillance radar consists of system of 4 planar phased antenna arrays. Each array covers one appropriate sector.

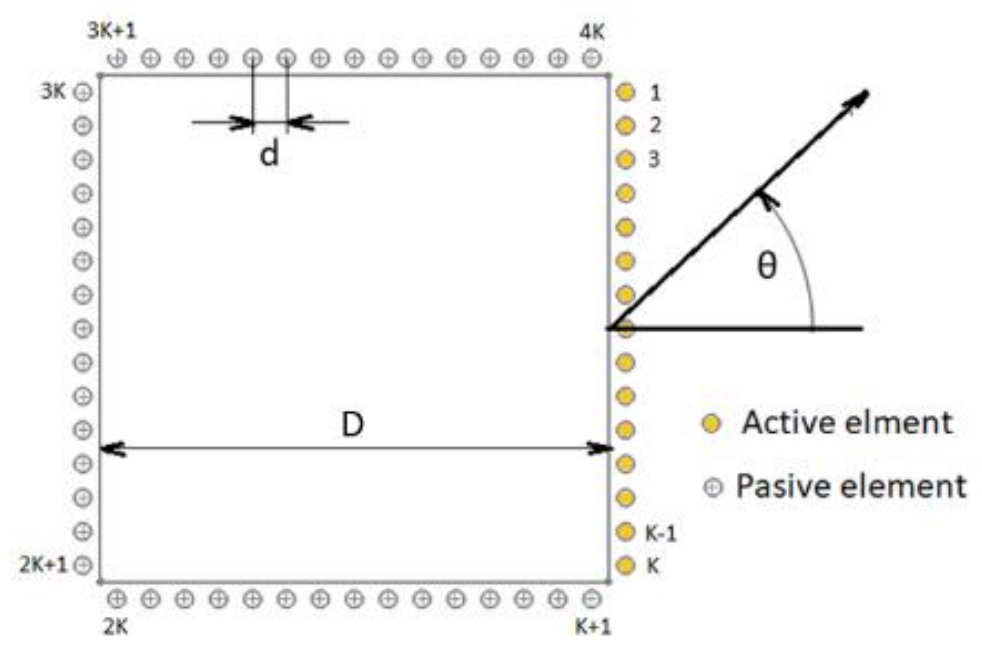

a) Square configuration

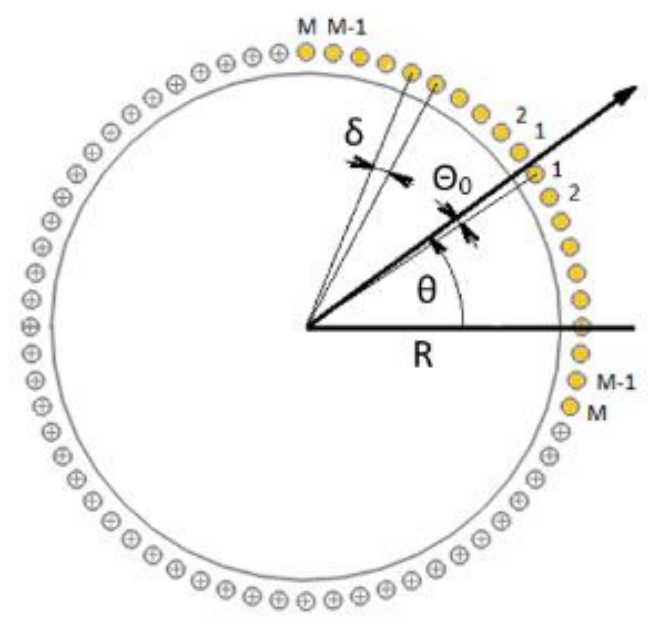

b) Circular configuration

Fig. 1. Configuration of four linear arrays and circular array for $360^{\circ}$. 
The beam is electronically steering by phase control of incident signals of array. The maximum deflection angle is $\pm 45^{\circ}$. The planar array can be considered as linear array of linear horizontal arrays.

The cylindrical antenna consists of linear array of circular horizontal arrays. The beam is steering in two steps. First the M elements left and right from main beam direction are switched on. Maximum percentage of active elements for one beam is $50 \%$. The other elements are off, means they has not influence to the pattern. This is happening when the element is electrically switched off, or his direction is such as not to affect to pattern of the analysing sector or is working on different frequency.

Second step in beam steering in cylindrical AESA is done by tuning phase of incident signals of array. The angle between the two elements in the array is small so the tuning is performed in a small range of several degrees. Thus, the pattern will have almost constant shape at all azimuth angles.

\subsection{Planar AESA}

The radar with a prismatic antenna is de facto system of 4 coordinated subradars with a planar AESA. Each of the 4 sub-radars covers its own sector. Although the number of transmitting beams can be arbitrary, in this paper we will analyse only sub radar with one beam.

A planar AESA can be considered as a linear array of $\mathrm{K}$ horizontal arrays with $\mathrm{M}$ elements. The total number of elements in one AESA is $\mathrm{M} * \mathrm{~K}$ and $4 * \mathrm{M} * \mathrm{~K}$ in the whole radar.

\section{$\underline{\text { Radiating element }}$}

In order to reduce the side lobes, the distance between the radiating elements should be less than $\lambda / 2$. Therefore, the radiating elements should be physically small. Suitable forms of radiating elements could be Vivaldi dipole [7]], printed dipole, small helicoidally antenna, slot dipole, etc. In this paper, an open-end waveguide is chosen, because the mutual impedance is small, the gain is significant and exact calculation pattern is available. The centre of frequency range is $3 \mathrm{GHz}$. Standard waveguide for the range $2.6 \mathrm{GHz}$ to $3.95 \mathrm{GHz}$ has dimensions: $\mathrm{a}=72 \mathrm{~mm} \mathrm{~b}=34 \mathrm{~mm}$. 
In order to enable distance between elements is less then $\lambda / 2$, the arrangement of shifted array of rectangular waveguide-ends is proposed, Fig. 2. The realized segment of two shifted linear arrays with 8 elements is shown on Fig. 3.

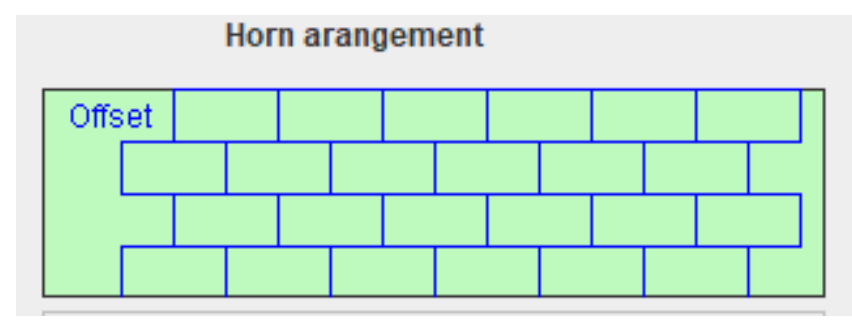

Fig.2. The arrangment of shifted array of rectangular waveguide open-end elements.

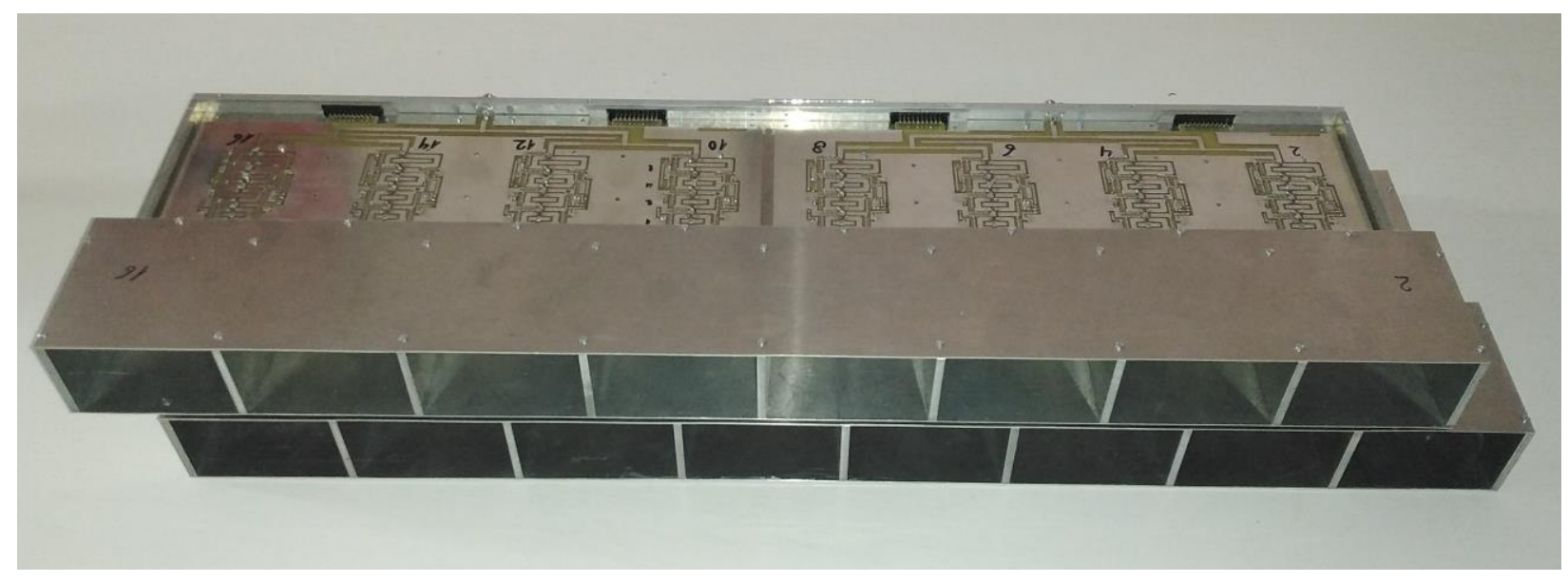

Fig. 3. Two shifted linear arrays of open-end waveguide for $3 \mathrm{GHz}$.

The arrangement with a circular open waveguide was also analysed, Fig. 4. The diameter of the waveguide for the band $26.5 \mathrm{GHz}$ to $3.3 \mathrm{GHz}$ is $\mathrm{d}=80 \mathrm{~mm}$. The analysis shows that the pattern of cylindrical AESA with uniform amplitude distribution has significantly smaller lateral lobes with circular open-end waveguide. The circular waveguide is additionally interesting because of possibility of crosspolarization, which enables the simultaneous use of two TR modules on two frequencies. Fig. 4. shows a scheme with a shifted arrangement of circular waveguides, and Fig. 5. shows the practical implementation of two horizontal arrays for IFF application. 


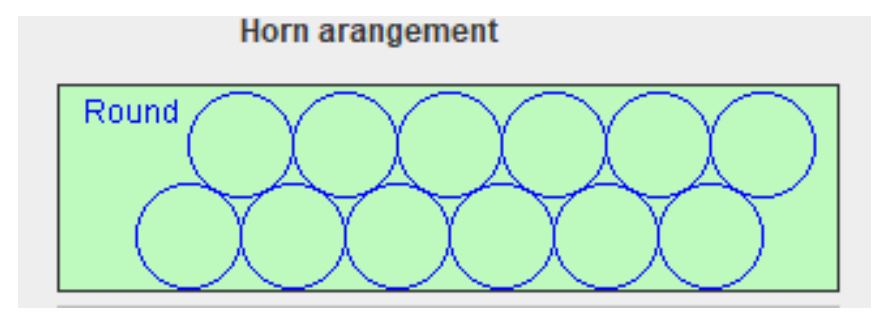

Fig. 4. The shifted horizontal arrangment of round waveguide open-end elements.

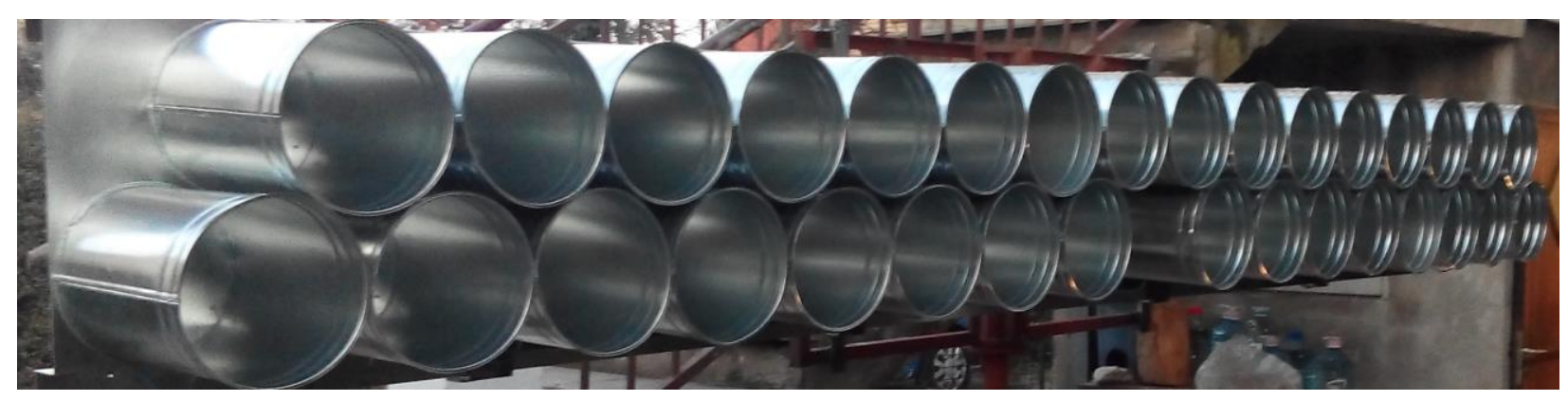

Fig. 5. Two arrays of 16 round open-end horn elements for IFF (1.030-1.090 GHz).

\subsection{Cylindrical AESA}

Radar with a cylindrical AESA can be considered as 2 coordinated sub radars with a semi-cylindrical AESA. Each of the 2 sub radars covers its own sector. In this article it is considered that each sub radar has only one main beam.

Cylindrical AESA is in fact a linear array of $\mathrm{K}$ horizontal circular arrays with $\mathrm{M}$ elements. The total number of elements in one semi-cylindrical AESA is $\mathrm{M} * \mathrm{~K}$ and in the whole radar $2 * \mathrm{M} * \mathrm{~K}$.

\section{Pattern calculation}

The pattern is calculated by summing the fields from each individual element using the basic formulas of analytical 3D geometry.

On the Fig.6. is shown the arrangement of the radiating elements in the $X Y Z$ Cartesian system. The line $s$, which has a direction $(0,0,1)$ and passes through the point $S(0, R, 0)$ is the axis of symmetry. The elements of the plane array at the points $\mathrm{A}_{\mathrm{mk}}\left(\mathrm{Ax}_{\mathrm{mk}}, \mathrm{Ay}_{\mathrm{mk}}, \mathrm{Az} \mathrm{z}_{\mathrm{mk}}\right)$ lie in the $\mathrm{YZ}$ plane: 


$$
\begin{array}{ll}
\mathrm{Ax}_{\mathrm{mk}}=0 & \\
\mathrm{Ay}_{\mathrm{mk}}=\text { yofset }+\mathrm{m} * \mathrm{dy} & \mathrm{m}=1: \mathrm{M} \\
\mathrm{Az}_{\mathrm{mk}}=\mathrm{k} * \mathrm{dz} & \mathrm{k}=1: \mathrm{K}
\end{array}
$$

The radiating open-end waveguides are directed in the direction $\mathbf{r}_{\mathbf{p m}}=(1,0,0)$.

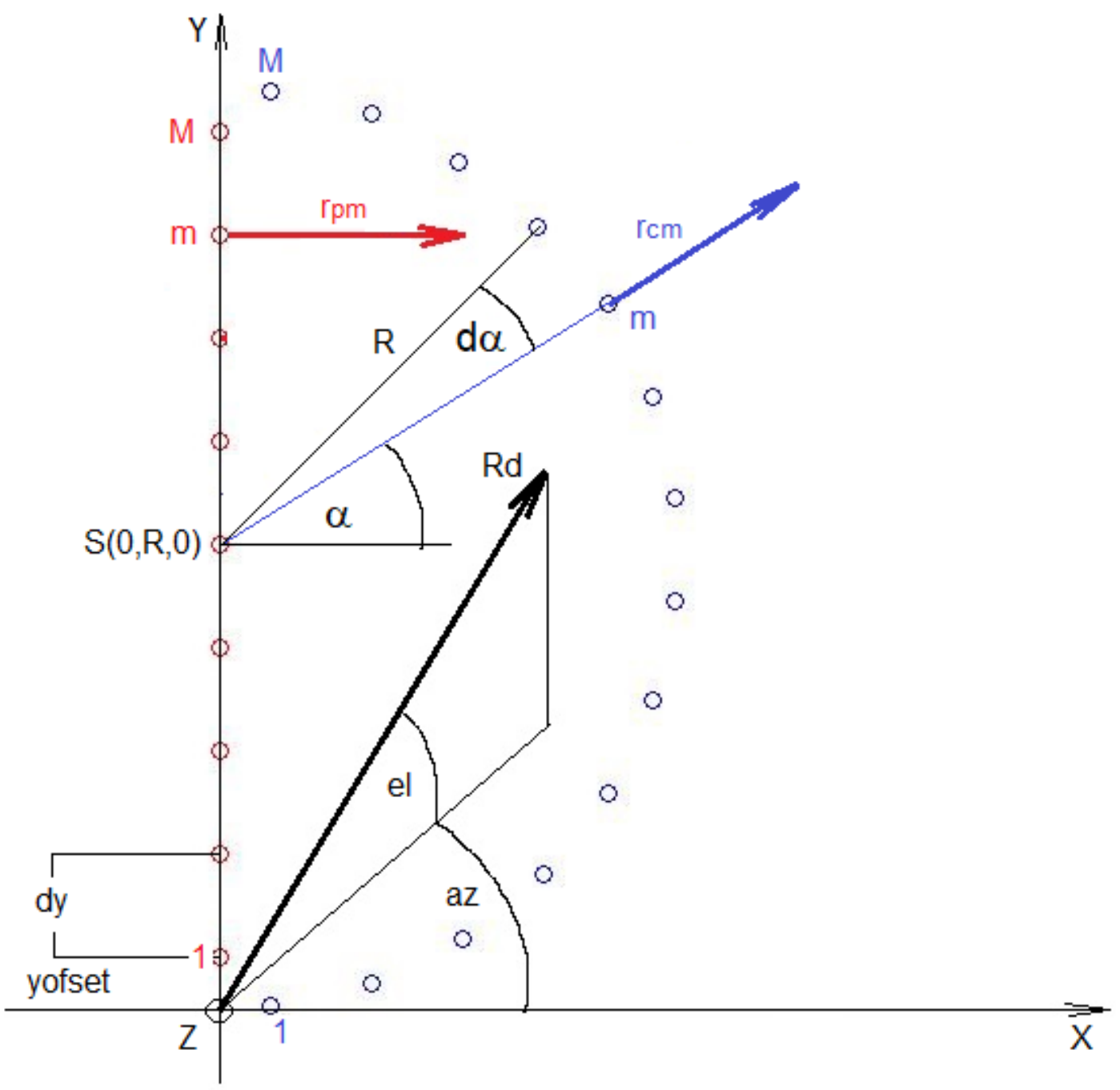

Fig. 6. Array arrangement.

The elements of the cylindrical AESA lie at the points $A_{m k}\left(A x_{m k}, A y_{m k}, A z_{m k}\right)$ on a half-cylinder whose axis of symmetry is line s:

$$
\begin{array}{ll}
\mathrm{Ax}_{\mathrm{mk}}=\mathrm{R} \cos (\alpha) & \\
\mathrm{Ay}_{\mathrm{mk}}=\mathrm{R}+\mathrm{R} \cos (\alpha) & \mathrm{m}=1: \mathrm{M} \\
\mathrm{Az} \mathrm{m}_{\mathrm{mk}}=\mathrm{k} * \mathrm{dz}, & \mathrm{k}=1: \mathrm{K}
\end{array}
$$


where

$$
\alpha=-\pi / 2-\mathrm{d} \alpha / 2+\mathrm{m} * \mathrm{~d} \alpha \quad \mathrm{m}=1: M
$$

The radiating open-end waveguides are directed in direction $\mathbf{r}_{\mathbf{c m}}=(\cos (\alpha), \sin (\alpha), 0)$.

The field is calculating in the direction of the $\mathbf{R}_{\mathbf{d}}$ vector. $\mathbf{R}_{\mathbf{d}}$ is created by rotating the vector $(1,0,0)$ for the angle az in the $X Y$ plane, and then for the angle el towards the $\mathbf{Z}$ axis. The vector $\mathbf{R}_{\mathbf{d}}$ has a direction

$$
\mathbf{R}_{\mathrm{d}}=(\cos (\mathrm{el}) * \cos (\mathrm{az}), \cos (\mathrm{el}) * \sin (\mathrm{az}), \sin (\mathrm{el}))
$$

The excitation signals are uniform for all radiating elements, so it can be assumed that their amplitude is 1 and phase 0 . The phase shifters introduce a delay $\Phi_{\mathrm{mk}}$, and programmable attenuators set the amplitude to $\mathrm{A}_{\mathrm{mk}}$. Additional phase shifts $\Psi_{\mathrm{k}}$ are used for horizontal arrays while forming multi-beams in the receiving mode. The plane $\Pi$ is normal to the direction $\mathbf{R}_{\mathbf{d}}$. The distance dist ${ }_{\mathrm{mk}}$ from the element at the point $A_{m k}$ to the plane $\Pi$ is calculated by the formulas of $3 \mathrm{D}$ analytical geometry. Propagating from the point $A_{m k}$ to the plane $\Pi$, the signal gets a phase shift of $2 \pi / \lambda *$ dist $_{\mathrm{mk}}$.

Complex signals in the plane $\Pi$ are

$$
\begin{aligned}
& \mathbf{p}_{\mathrm{mk}}=\mathrm{A}_{\mathrm{mk}}\left(\cos \left(\varphi_{\mathrm{mk}}\right)+\mathrm{j} \sin \left(\varphi_{\mathrm{mk}}\right)\right) \operatorname{EL}\left(\mathrm{az} \mathrm{mk}_{\mathrm{m}}, \mathrm{el}_{\mathrm{mk}}\right) \\
& \varphi_{\mathrm{mk}}=\Phi_{\mathrm{mk}}+\Psi_{\mathrm{k}+} 2 \pi / \lambda * \mathrm{dist}_{\mathrm{mk}}
\end{aligned}
$$

where

$\mathrm{EL}\left(\mathrm{az} \mathrm{mk}_{\mathrm{mk}}, \mathrm{el}_{\mathrm{mk}}\right)-$ normalized radiation function of the radiating element in function of $a z_{\mathrm{mk}}$ and $\mathrm{el}_{\mathrm{mk}}$.

$a z_{m k}$ - difference between the horn orientation angle and the given azimuth direction

$\mathrm{el}_{\mathrm{mk}}$ - difference between the horn orientation angle and the given elevation direction.

The cumulative signal in the direction $\mathbf{R}_{\mathbf{d}}$ is

$$
\mathbf{P}=\Sigma \mathbf{p}_{\mathbf{m k}} \quad \mathrm{k}=1: \mathrm{K}, \mathrm{m}=1: \mathbf{M}
$$


The calculation process is performed in the following steps:

- Based on the given AESA dimensions and horn dimensions, the number and position of the radiating elements are calculated

- The plane $\Pi$ is created normal to the radiation direction $\mathbf{R}_{\mathbf{d}}$ in point $\mathrm{O}(0,0,0)$

- The distance dist $_{\mathrm{mn}}$ from each element to the plane $\Pi$ is calculated

- The gain of the radiating element $\mathrm{G}\left(\mathrm{az} \mathrm{z}_{\mathrm{mk}}, \mathrm{el}_{\mathrm{mk}}\right)$ is calculated

- The complex sum by equation (11) is calculated.

In order to obtain the maximum antenna's gain in the $\mathbf{R}_{\mathbf{d}}$ direction, the fields of each element must be in phase in the plane $\Pi$. To obtain a special shape of the main beam or to suppress the side lobes, optimized amplitude $A_{m k}$ and a phase $\Phi_{m k}$ distribution should be used.

Directivity is calculated by an approximate formula:

$\operatorname{Dir}=41253 /\left(\theta_{3 \mathrm{dbh}} * \theta_{3 \mathrm{dbv}}\right)$

where

$\theta_{3 \mathrm{dbh}}$ is $3 \mathrm{~dB}$ width angle in the horizontal plane,

$\theta_{3 \mathrm{dbv}}$ is $3 \mathrm{~dB}$ width angle in the vertical plane.

For efficient and visual comparative analysis, a special program in Java was developed with the GUI shown in Fig. 7.

The right part of the window is used to define the type and dimensions of the AESA. Other inputs are also specified. There are two graphic components in the middle part for visual illustration of created AESA. The lower part of the middle of the window is used to define the graphic properties of pattern display.

The pattern display is in the left part. The basic representation of the pattern is in the function of azimuth or elevation. It enables a precise display of the main beam width and the level of the side lobes, as in Fig. 10. In order to get a complete insight into the characteristics of the pattern, a quasi-3D display is used, as in Fig. 13.-down. The azimuth is on abscissa and elevation on ordinate. The level is shown by shade of red. The $3 \mathrm{~dB}$ zone is shown in blue, and the maximum radiation is marked with a yellow dot. This type of quasi-3D display emphasizes well beam deformation. 
However, it produces a deformation when transforming 3D pattern from spherical coordinated system to rectangular 2D azimuth-elevation system. It gives a quite realistic picture at low elevation, but a deformation at high elevation level is significant like on Fig. 22. left.

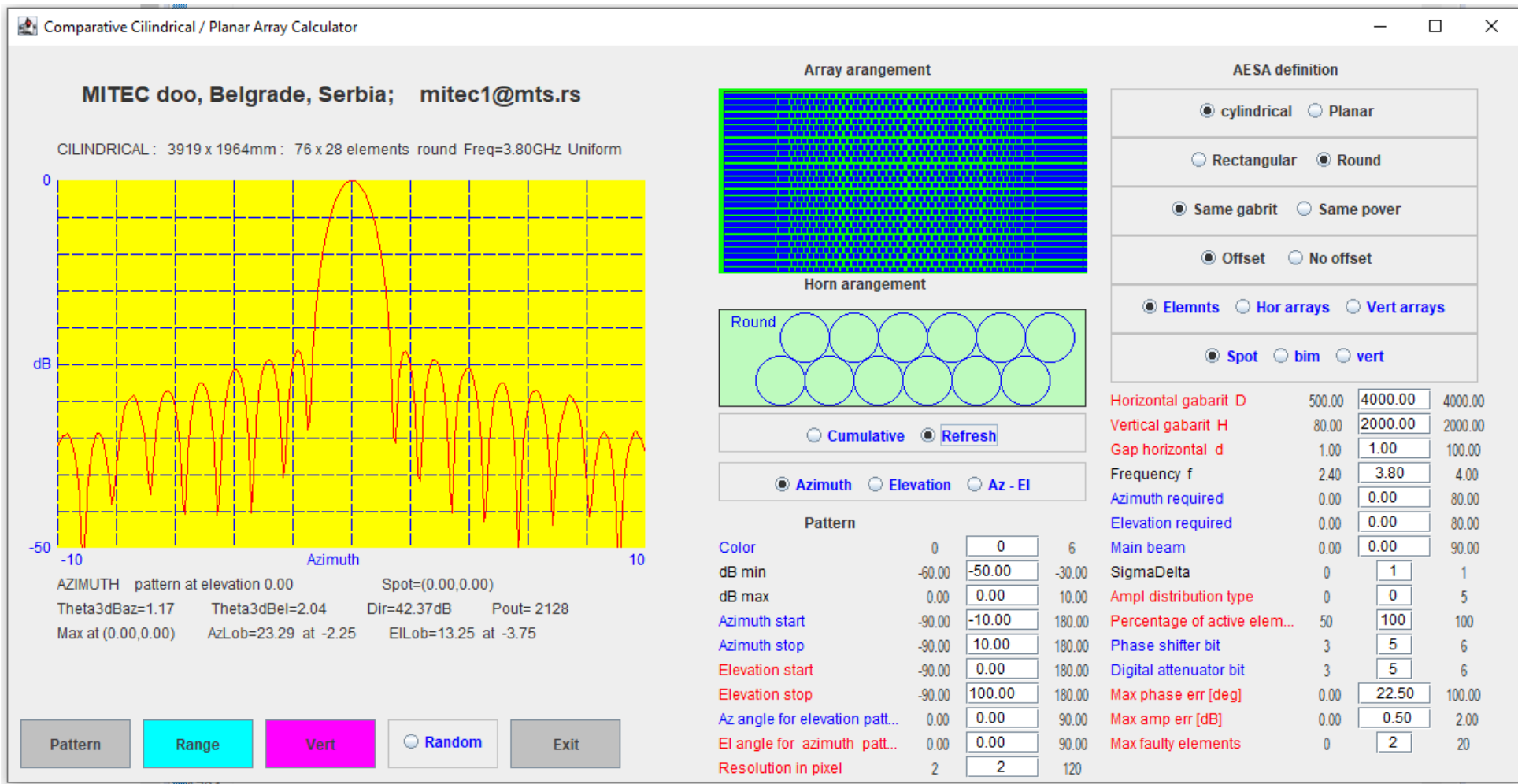

Fig. 7. Comparative Cylindrical / Planar Calculator GUI.

For a more realistic representation of the beam deformation at hight elevation angles, a quasi-3D representation is used, as in Fig. 22. on the right. A spherical sector whose axis coincides with the direction of the main beam is observed. The angle of the spherical sector is $20^{\circ}$ for all images in this article. The projection of the sector to the tangential plane at the point of the sector's centre is shown. This quasi$3 \mathrm{D}$ view is quite precise in the middle of the sector.

Some alpha numerical results are also displayed. Since they are visible in most images, a description of the output results is given here to make it easier to follow the images.

The line above the graph shows the AESA structure:

\section{- CIRCULAR / PLANAR array \\ cylindrical or planar type AESA}

- width by height in $\mathrm{mm}$

- number $\mathrm{M}$ of elements in horizontal array, or number of horizontal arrays 
- number K of horizontal arrays or number of elements in vertical arrays

- rect / round

type of horn

- Freq

working frequency

- Uniform / Optimized uniform or optimized amp. distribution.

First row below the graph shows type of pattern presentation:

- AZIMUH-ELEVATION

- SECTOR

- AZIMUH

- ELEVATION

- AZ0-EL0
- quasi 3D az-el pattern view

- normal view to the spherical sector around the main beam

- conical section, $\theta=$ const

- flat section with the vertical plane $\Gamma$, Fig. 11. right

- flat section with the plane $\Lambda$, as on Fig.11. left

The plane $\Lambda$ is perpendicular to vertical plane $\Gamma$. It passes through the coordinate origin and contains the vector $\mathrm{p}$ of main beam's direction. From this section, a 3dB angle in the horizontal plane was obtained to calculate the gain.

The middle row below the figure shows the width of the angle of the main beam in azimuth and elevation, directivity and radiated power.

The third row below provides data of the maximum side lobe.

\section{Beam forming network}

The speed of observation is important for surveillance radar. Modern surveillance radar in the multi-beam reception mode MBM scans the complete elevation with one transmit pulse. However, this affects the complexity of the radar construction. To assess the performance and complexity of the radar, the following will be analysed in more detail: single beam receive mode SBM network, MBM in Array of arrays configuration and MBM in Array of elements configuration in both analogue $\mathrm{ABF}$ and digital beam forming $\mathrm{DBF}$.

\subsection{Single beam receiving mode $S B M$}

\subsubsection{Planar AESA}

The block diagram of a single beam forming is shown in Fig. 8. The transmitting signal $\mathrm{Tx}$ is evenly divided into $\mathrm{N}=\mathrm{M} * \mathrm{~K}$ outputs, for each radiating element. The required phase and amplitude distribution for electronic steering is performed in the $\Phi /$ Att digital phase shifter and attenuator block. The signal is amplified in the PA power amplifier. 
It is assumed that the number of TR modules is equal to the number of radiating elements. On the receiving side, the signal is limited in Limiter and amplified in the LNA. Signals from all radiating elements are summed in sumator.

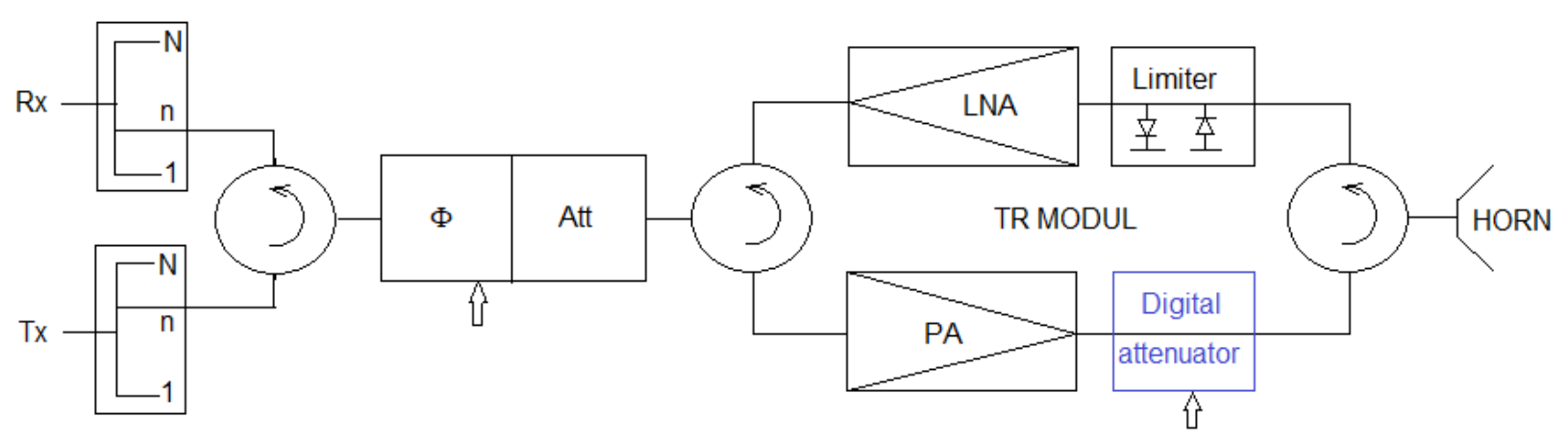

Fig. 8. Block schema for Single Beam Forming SBM.

\subsubsection{TR module}

The functional diagram of the TR module is shown on the right part of Fig. 8. The functional circulators can be ferrite circulators or PIN diode switch. If a beam shape with a uniform amplitude distribution is acceptable, a Digital attenuator block is not required. The signal level on the $\Phi /$ Att block is low, so both phase shifters and attenuators can be for low RF levels. If there is no Digital attenuator block, the PA power amplifier must be linear. This is certainly an aggravating requirement, as the maximum output power and $\eta$ of the amplifier are reduced. It is desirable that the PA works in saturation, then the output power is less dependent on temperature and frequency. Therefore, it is more advantageous that digital attenuator for Tx mode be behind the PA. On the other hand, the Digital attenuator must be designed for high output powers in accordance with the output power of the PA. Adequate cooling must also be provided.

Monolithic TR modules are suitable due to the small deviation of characteristics from piece to piece. Any deviation affects the AESA pattern.

\subsubsection{Digital Phase Shifter and Digital attenuator system}

Digital Phase Shifters should be adapted to the signal level and frequency range. The number of control bits should be adjusted to the sensitivity of the pattern. 


\subsubsection{RF Combiner and Divider Network}

Amplitude and phase distribution are achieved by an RF distribution network. It is consisting of dividers and combiners. The shape of the network is arbitrary, and here it is assumed to be in the shape of a binary tree. This shape provides the optimum in terms of cable length and divider uniformity. The physical length of the cables at the same level of nodes is the same, so it is easier to maintain a uniform phase distribution. This is especially pronounced in long-cable networks, such as at low-frequency radar. If the number of ending nodes TR modules is not $2^{\mathrm{n}}$, unused nodes should be closed with a matched termination, so that the input impedance of the divider is correct, and the distribution is uniform. In Fig. 8. the transmitting and receiving RF distribution networks are shown separately. In practical implementation, the physically same transmission and reception network can be used, especially in AESA with a raspberry number of elements.

The attenuation in an ideal distribution network is $10 \log \mathrm{N}$, where $\mathrm{N}$ is the number of outputs. In AESA with a large number of elements, the attenuation in the network can be large. For example, for a circular AESA with a diameter of $4 \mathrm{~m}$ and a height of $2 \mathrm{~m}$ at $3 \mathrm{GHz}$, the number of elements is of the order of 10000 , and the attenuation is $40 \mathrm{~dB}$. If the PA in TR module is a single-stage output power of $20 \mathrm{~W}$ and a gain of $15 \mathrm{~dB}$, the required input level in the TR for saturation is about $1 \mathrm{~W}$. Then a level of $70 \mathrm{dBm}$ or $10 \mathrm{KW}$ is required at the Tx input. This is certainly unfavourable, so the solution should be sought on the introduction of line amplifiers at several levels in the distribution network. If the network is used for reception mode, each line amplifier requires a circulator at the input and output to separate the transmitting from the receiving signal.

\subsubsection{Digital and Pulse wire Network}

Although not presented on Fig. 8. a very extensive wire network is required. For each TR module and $\Phi /$ Att block, the appropriate number of DC voltages are required. The triggers for drain and gate of PA, PIN switch command, multi-bit signal for setting phase shift and attenuator attenuation, signals for status indication, 
etc. are required. Network complexity is directly proportional number of TR modules.

\subsubsection{Cylindrical AESA}

The block diagram for a single beam mode (SBM) cylindrical AESA is shown on Fig. 9.

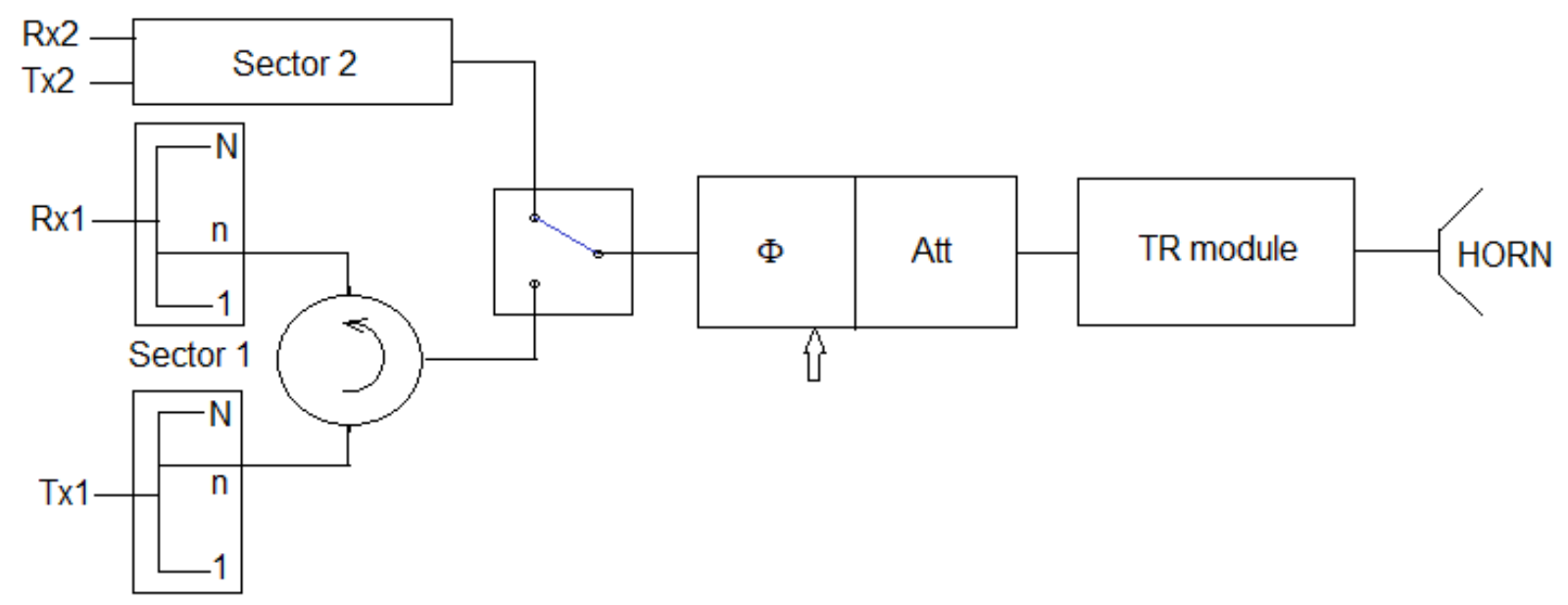

Fig. 9. Block schema for Single Beam Forming SBM for cylindrical AESA.

Most elements in the SBM configuration of cylindrical AESA are the same as for planar AESA. While in a planar AESA each TR module is used in only one beam, in a cylindrical AESA each TR module is used in one of two beams. Therefore, a switching block is required to connect the TR module to the appropriate $\mathrm{RF}$ distribution network. It can be physically located in the TR module, or in the RF distribution network, or as a standalone component. The switch must be absorptive type so as not to affect the impedance of the RF distribution network.

The number of inputs $\mathrm{N}$ in the divider and combiner is equal to the total number of TR modules, but only half is active. It is immediately apparent that the RF distribution network is more complicated with cylindrical AESA, although the total number of $\mathrm{N}$ elements is not greater than one of planar AESA.

The same open-end waveguide horns, and in the same arrangement, are used in both cilindrical and the planar AESA. Due to the unniformn spatial orientation of the horns in the cylindrical AESA there is a passive amplitude distribution although the excitation signals are uniform. The pattern of a cylindrical array of round wavguide 
horn with uniform distribution is interesting, Fig. 10. The differnce bitween side lobe level of rectangular horn array (red) and round horn array (blue) is 10dB.

CILINDRICAL : $3919 \times 1964 \mathrm{~mm}: 76 \times 28$ elements round Freq=3.80GHz Uniform

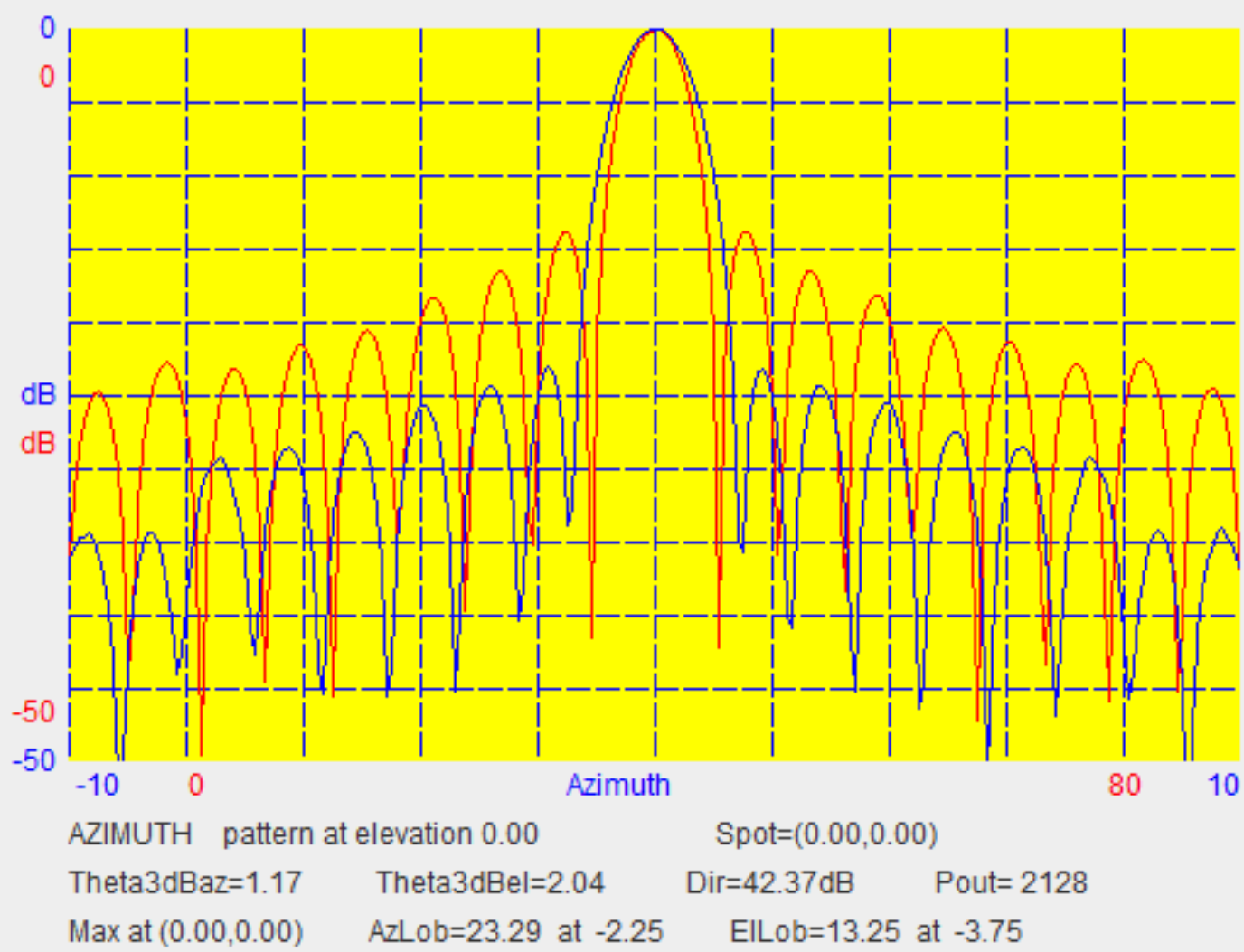

Fig. 10. Cilindrical AESA with uniform amplitude distribution: red - rectangular waveguide, blue - round waveguide.
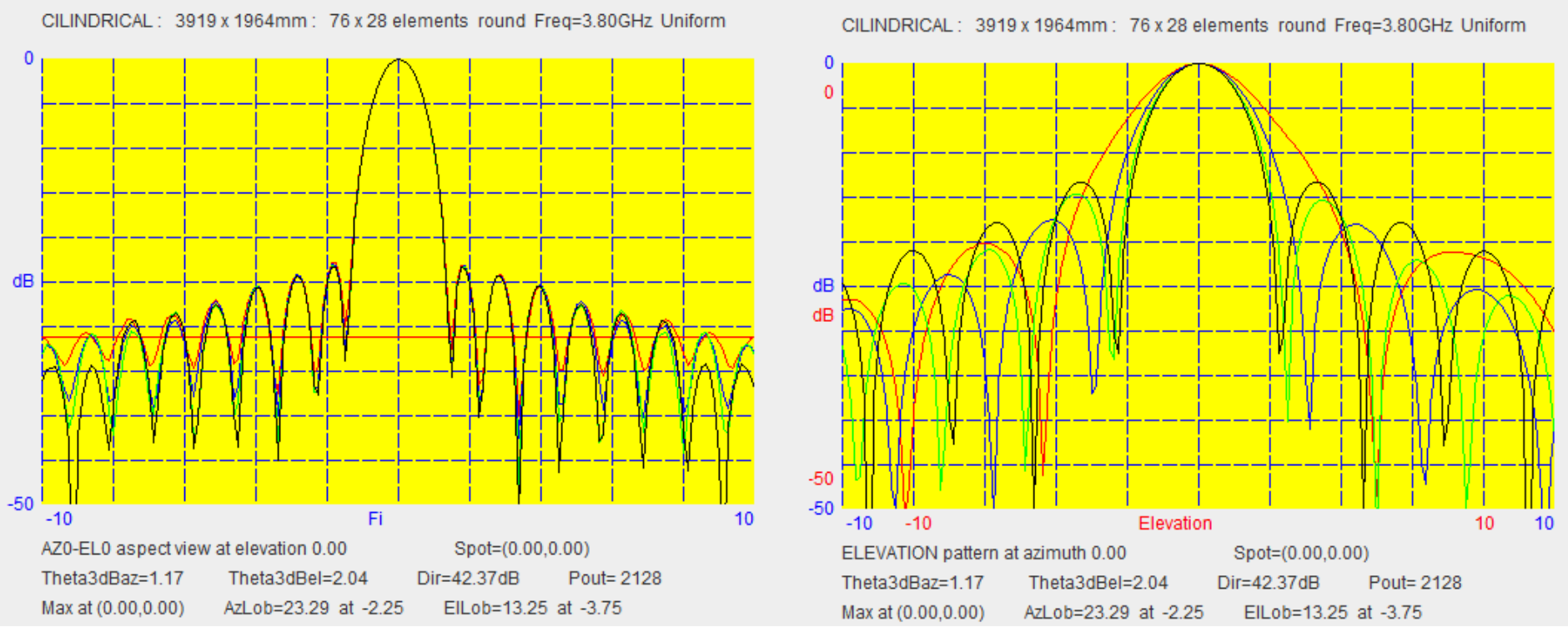

Fig. 11. Spreading of beam of cilindrical AESA as a function of elevation: red - 60, blue - 40, green -20 and black - 0 degrees. 
The beam of cylindrical AESA spreads in vertical plane while scanning in elevation, figure 11 . right. The gain decreases from $42.37 \mathrm{~dB}$ at $0^{\circ}$ to $41.12 \mathrm{~dB}$ at $60^{\circ}$. The beam width in horizontal flat section is almost constant, figure 11. left.

\subsection{Multiple beam receive mode MBM in Array of arrays configuration}

Single beam receive mod is useful for targeting mode or for weather radar. It is not practical for 3D surveillance radar, because the surveillance time is too long. For efficient 3D scanning, MBM mode is used to scan complete elevation angle with one Tx pulse. Therefore, we will deal in more detail with MBM reception mode because it directly affects the surveillance time and the complexity of the construction.

The basic idea is to keep all the functionalities of the rotating AESA, and to introduce electronic azimuth scanning instead of mechanical rotation. This leads to the concept of AESA as a series of horizontal arrays. The logic scheme is shown in Fig. 12. Modules that are not needed for the rotating AESA are marked in blue, and the block used only in the cylindrical AESA is marked in red.

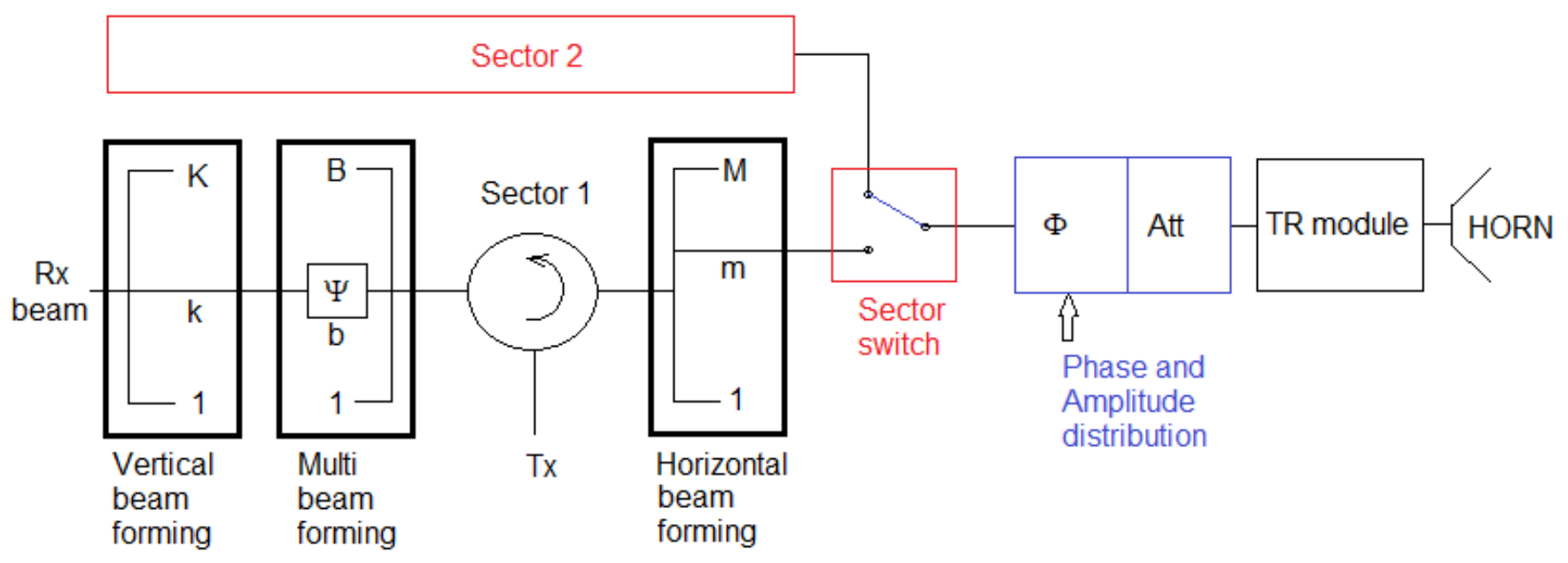

Fig. 12. Analogue Beam Forming with Array of arrays configuration.

In the transmit mode, the transmitting Tx signal is fed thru the circulator to the Horizontal beam forming distributor, which distributes the excitation signal evenly and in phase to all radiating elements. In the Phase and Amplitude distribution 
block, the necessary phase distribution is performed using digital phase shifters to steer the beam in the desired direction.

The use of digital attenuators achieves the required amplitude distribution for pattern formation. It is desirable to radiate as much power as possible. If a high level of lateral lobes is acceptable, the uniform amplitude distribution is best suited for this. Then digital attenuators are not required. The beam of rotating AESA is in the direction normal to the array plane, so phase shifters are not required.

In the radar with 4 planar AESAs, each TR module participates in only one beam. However, in a cylindrical AESA, each TR module can participate in the formation of one of the two beams. Since two beams are oppositely oriented, each beam is formed by half of the radiating elements from the corresponding halfcylinder. This requires a Sector switch block to join the TR block to corresponding sector. The Sector switch block consists of a set of RF switches. The fine steering of the beam, of only a few degrees, is done by means of phase shifters.

The Phase and Amplitude distribution block is used to form the beam in the horizontal plane. The signals from the elements of horizontal array are summed in the Horizontal beam forming block. It is in fact an RF combiner with M inputs and one output. The phase and amplitude distribution are uniform. Each horizontal array has its own Horizontal beam forming block, so the required number of blocks is equal to the number of horizontal arrays $\mathrm{K}$.

The set of B vertical arranged receiving beams are formed for MBM mode. The target's elevation can be determined comparing the receiving signal in them. To enable multi beam mode, $\mathrm{K}$ dividers are required, one for each horizontal array. The dividers have B outputs, one for each beam. Additional phase shifters $\Psi$ are required for to achieve elevation steering.

The phase shifter $\Psi$ can also be physically in the next RF combiner Vertical Beam Forming which has $\mathrm{K}$ inputs. One combiner is for each beam, so the total number of combines is $\mathrm{B}$. 
Table T1. Hardware complexity of AESA Array of horizontal arrays configuration.

\begin{tabular}{|c|c|c|c|c|}
\hline & Cylindrical & $\begin{array}{c}\text { Planar } \\
\text { (same size) }\end{array}$ & $\begin{array}{c}\text { Planar } \\
\text { (same power) }\end{array}$ & $\begin{array}{c}\text { Rotating } \\
\text { (one AESA) }\end{array}$ \\
\hline Dimension & $4 \times 2 \mathrm{~m}$ & $4 \times 2 \mathrm{~m}$ & $3.14 \times 2 \mathrm{~m}$ & $4 \times 2 \mathrm{~m}$ \\
\hline K horizontal arrays & 54 & 54 & 54 & 54 \\
\hline $\begin{array}{l}\text { M elements in } \\
\text { horizontal array }\end{array}$ & 86 & 54 & 44 & 54 \\
\hline $\mathrm{B}$ receive beams & 12 & 12 & 12 & 12 \\
\hline Nh horns, TR, atten & $\begin{array}{c}\mathrm{K} \times \mathrm{M} \times 2= \\
9288\end{array}$ & $\begin{array}{c}\mathrm{K} \times \mathrm{M} \times 4= \\
11664\end{array}$ & $\begin{array}{c}\mathrm{K} \times \mathrm{M} \times 4= \\
9504\end{array}$ & $K \times M=2916$ \\
\hline Nph phase sifters & $2 \mathrm{~K}(\mathrm{M}+\mathrm{B})=10584$ & $4 \mathrm{~K}(\mathrm{M}+\mathrm{B})=14256$ & $4 \mathrm{~K}(\mathrm{M}+\mathrm{B})=11616$ & $\begin{array}{c}\mathrm{B} \times \mathrm{K} \\
648\end{array}=$ \\
\hline Nsw switches & 9288 & 0 & 0 & 0 \\
\hline $\begin{array}{l}\text { Ncm comb, M } \\
\text { inputs }\end{array}$ & $\begin{array}{l}54 \text { with } 172 \\
\text { input }\end{array}$ & 54 with 54 inputs & 54 with 44 inputs & $\begin{array}{l}54 \text { with } 54 \\
\text { inputs }\end{array}$ \\
\hline $\begin{array}{l}\text { Nd dividers with B } \\
\text { outputs }\end{array}$ & 86 with 12 & 54 with 12 & 44 with 12 & 54 with 12 \\
\hline Nck comb, K inputs & 12 with 54 inputs & 12 with 54 inputs & 12 with 54 inputs & $\begin{array}{c}12 \text { with } 54 \\
\text { inputs }\end{array}$ \\
\hline Mechanical rotator & No & No & No & Yes \\
\hline Rotary joint & No & No & No & Yes \\
\hline
\end{tabular}

In table $\mathrm{T} 1$ is given the example of quantitative analysis of the system from Figure 12. The quantities are shown for complete cylindrical AESA, four-side prismatic AESA of the same installed RF power as cylindrical AESA, four-side prismatic AESA of the same size as cylindrical AESA and one rotating flat AESA of the same dimensions as cylindrical. In all cases, rectangular horns measuring 72 x 36 $\mathrm{mm}$ are used in a shifted arrangement. It is assumed that there are $\mathrm{B}=12$ beams in the vertical plane.

The red fields in the table indicate items that greatly increase the complexity of the construction. Highly suitable items are marked in green. The rotating planar AESA is much simpler in terms of RF beam forming circuits. On the other hand, a mechanical rotation system and a rotary joint are required.

All 3 non-rotating systems are similar in complexity. All variants require a significant number of TR modules, phase shifters and digital attenuators. The most complex of them is the radar with cylindrical AESA. It has an additional system of large number of RF switches. In addition, RF splitters with 172 inputs can be complicated in electronic and spatial terms. 
Quantitative analysis of the MBM mode in the Array of horizontal arrays configuration indicates spatial limitations when scanning.

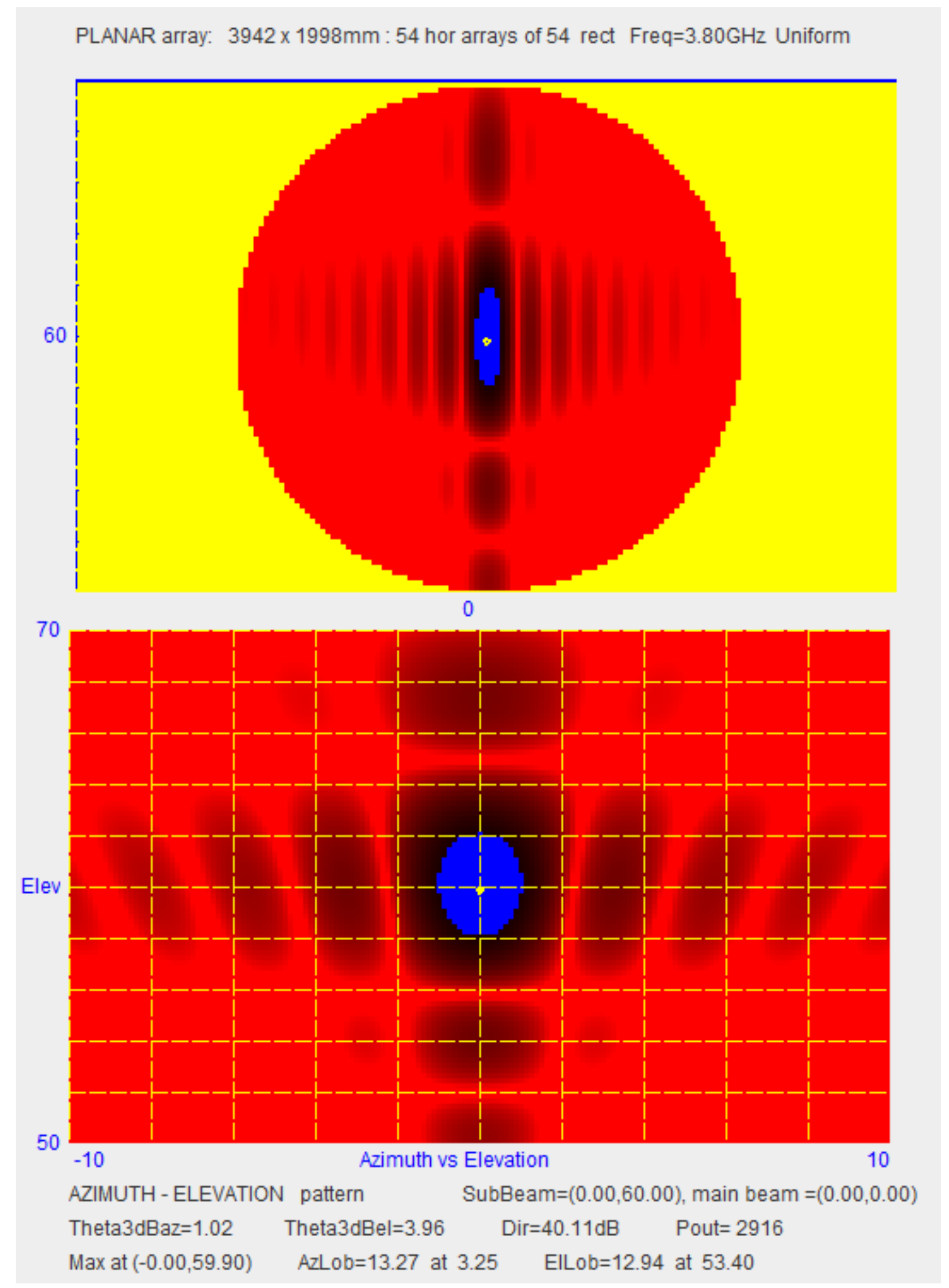

Fig. 13. Pattern of MBM for planar AESA at azimuth $0^{\circ}$ and elevation $60^{\circ}$. It is also the pattern for rotating AESA. 


\section{PLANAR array: $3942 \times 1998 \mathrm{~mm}: 54$ hor arrays of 54 rect Freq=3.80GHz Uniform}
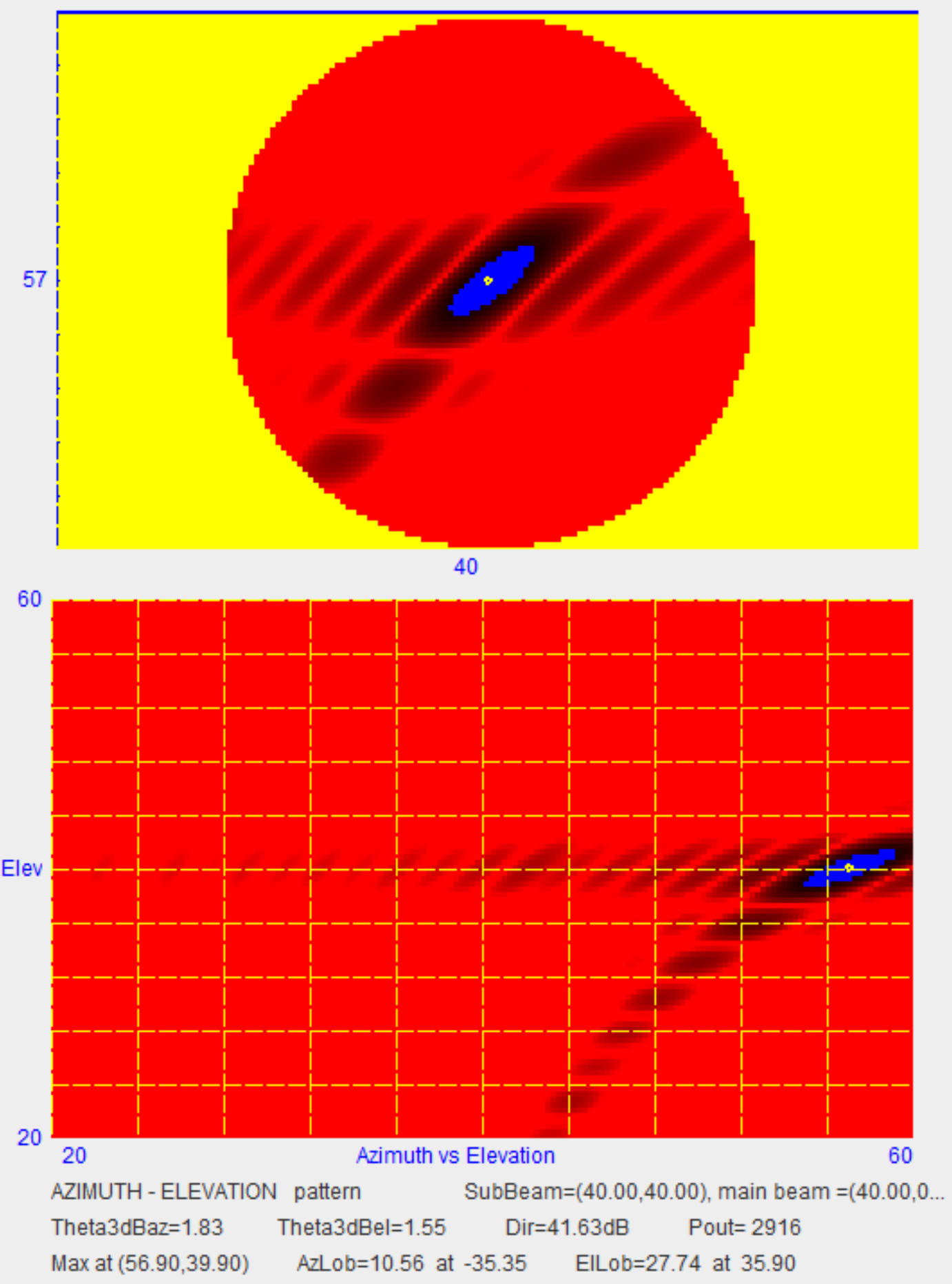

Fig 14. Beam moving error of planar array while multiple beam forming in array of arrays configuration.

The pattern, Fig. 13, for a planar AESA (and for a rotating AESA) at an azimuth of $0^{\circ}$ is correct even for an elevation angle of $60^{\circ}$. During the azimuth steering, the beam spreads and deforms, the side lobes increase and the error in the 
sub-beam direction occurs. The pattern of the receiving sub-beam for the azimuth direction of $40^{\circ}$ and elevation of $40^{\circ}$ is shown on the Fig. 14. The error in the azimuth direction of $16.9^{\circ}$ occurs. The main beam deformation is visible on both up and down part of figure. The error is predictable but unacceptable for modern radar. Obviously, there is a spatial limit in the use of a planar AESA in MBM receiving mode.

CILINDRICAL : $3996 \times 1998 \mathrm{~mm}: 54$ hor arrays of 86 rect Freq $=3.00 \mathrm{GHz}$ Uniform

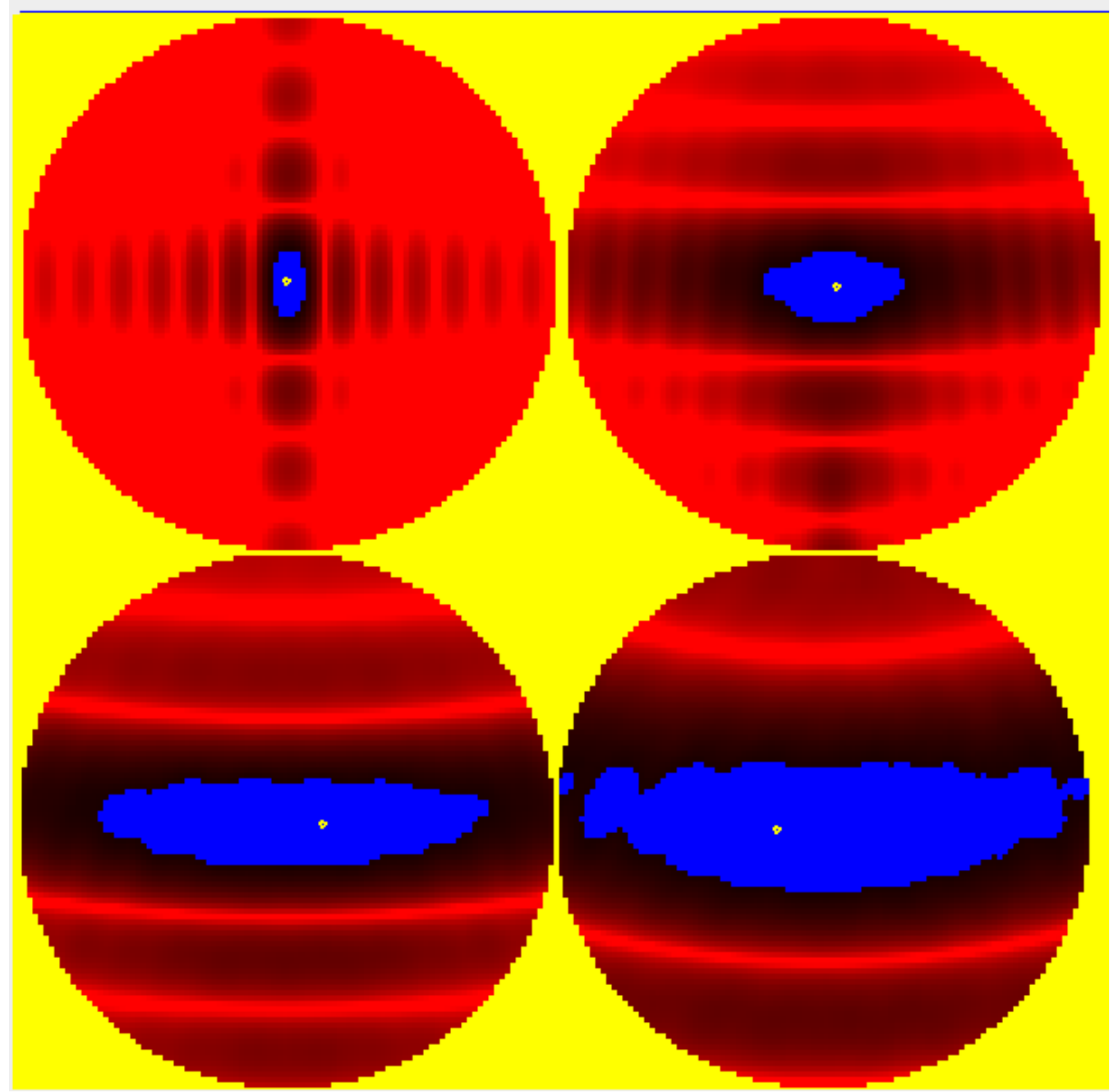

Fig 15. Pattern distorsion of cilindrical array in MBM mode in Array of arrays configuration. 
It can be seen from Fig. 15. that distortion also occurs with a cylindrical AESA. The main transmitting beam is directed to an elevation of $0^{\circ}$. As the elevation angle of the receiving subbeams increases, the beam spreads drastically in horizontal plane. The beam shape is shown for the elevation of $0^{\circ}, 20^{\circ}, 40^{\circ}$ and $60^{\circ}$.

Fig.16. shows more precisely the shape of the receiving subbeam for diferent elevation values. The beam width in horizontal plane spreads from $1.24^{\circ}$ at elevaton of $0^{\circ}$ to $19.4^{\circ}$ at elevation $40^{\circ}$. The subbeam expands unacceptably much at about 30 . Although, unlike planar AESA, there is no subbeam's direction error, there is limit of use of cylindrical AESA in MBM receive mode.

CILINDRICAL : $3996 \times 1998 \mathrm{~mm}: 54$ hor arrays of 86 rect Freq=3.00GHz Uniform

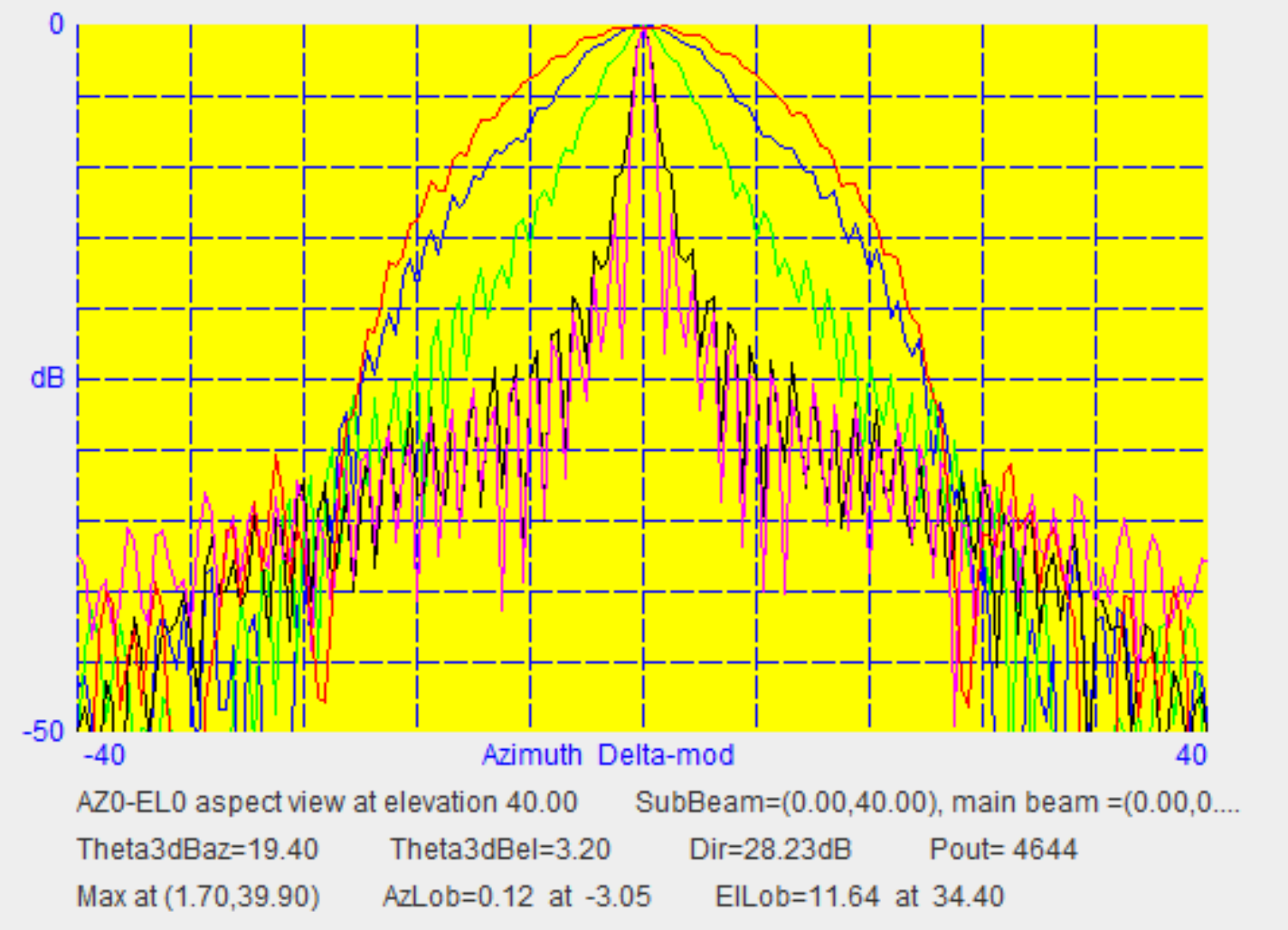

Fig. 16. Cylindrical array of horizontal arrays MBM for elevation: red - 40-crvena, blue - 30, green - 20, black - 10, magenta - 0 degrees.

The Array of horizontal arrays configuration for MBM is relatively simple to implement. It is also easy to calculate the required phase and amplitude distribution to obtain the desired beam shape. On the other hand, severe beam deformations significantly limit the spatial range of application. 


\subsection{MBM for Array of elements configuration}

Beam deformations in MBM mode can be significantly reduced in the Array of elements configuration. On Fig. 17. is shown a pattern for a planar AESA under the same conditions as for the Array of array configuration whose pattern is shown in Fig. 14. The difference in quality in favour of the Array of elements is obvious. Fig. 18. shows that a quality pattern can be obtained for cylindrical AESA even for an elevation angle of $60^{\circ}$.

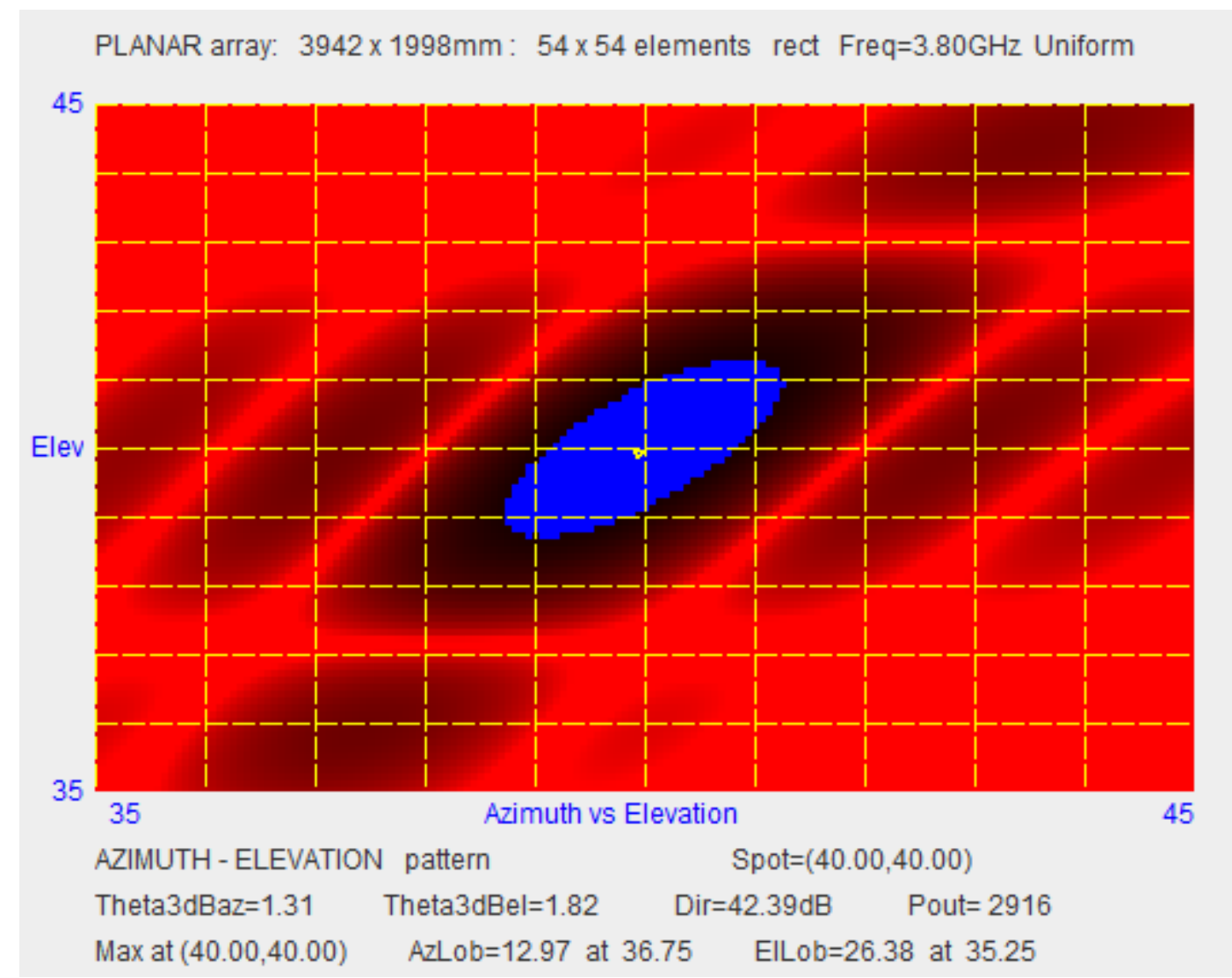

Fig. 17. Pattern of Array of elements MBM for planar AESA.

The block diagram of the Analog Beam Forming ABF system is shown in Fig. 19. Although the block diagram looks simpler, the construction is technically very complex. 
CILINDRICAL : $3996 \times 1998 \mathrm{~mm}: 86 \times 54$ elements rect Freq $=3.80 \mathrm{GHz}$ Uniform

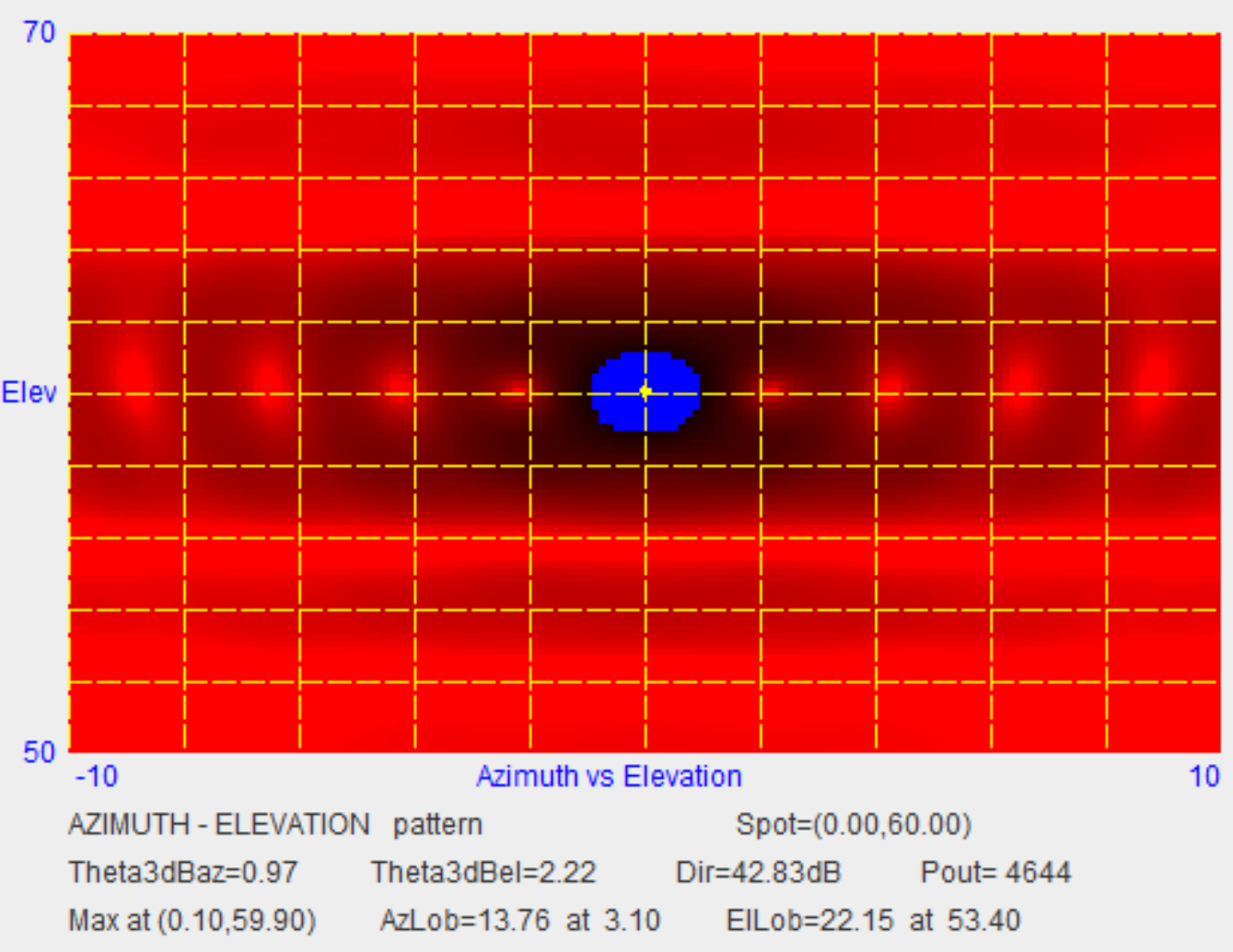

Fig. 18. Pattern of Array of elements MBM for cylindrical AESA.
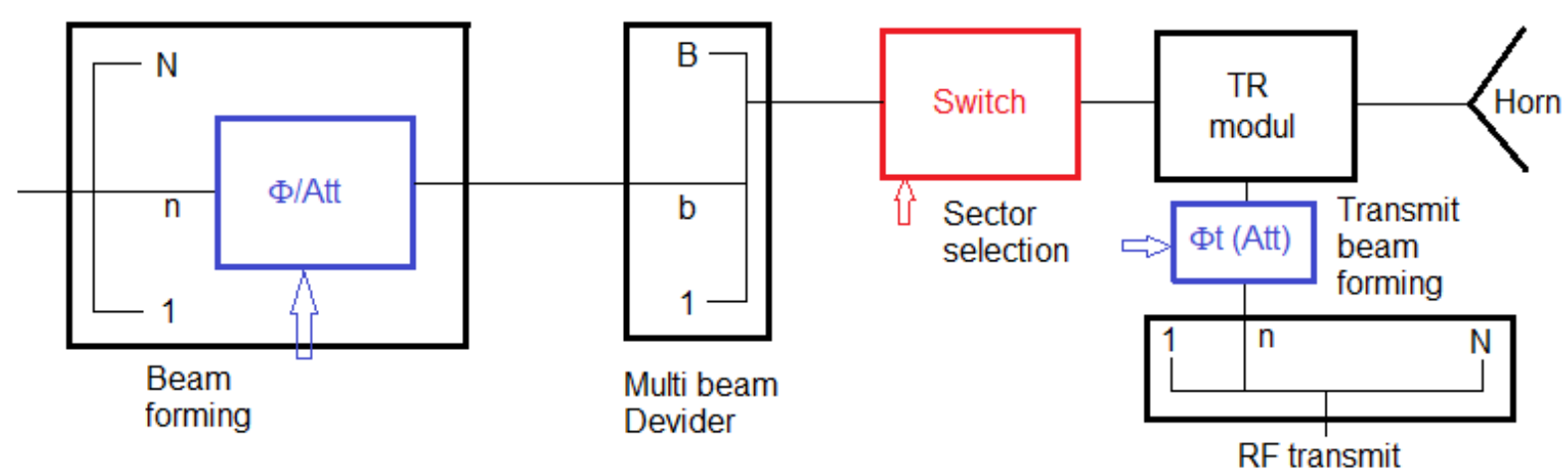

Fig. 19. ABF with Array of elements.

The transmission part is the same as in the Array of arrays configuration, except that the Transmit beam forming block consists of physically different phase shifters and attenuators block from the receiving one. A cylindrical AESA requires a Switch block to switch the element to one of two sectors. Behind each radiating element comes an RF divider with B outputs, for each of the B receiving beams. 
A Beam forming block is required for each receiving beam. It is a very complex block: it has $\mathrm{N}=\mathrm{M} * \mathrm{~K}$ inputs. For each input there is $\Phi /$ Att block for steering and shaping the beam. There is RF combiner with $\mathrm{N}$ inputs at the output of the Beam forming block.

In addition to the large number of elements in the Array of elements configuration, there are also problems with placing blocks in space. It is difficult at high frequency, where the dimensions of the horn are small, to provide space for 12 connectors for 12 receiving beams. Also, the Beam forming block has a huge number of inputs, so it is not easy to organize a network of cables and dividers for B Beam forming blocks.

Table T2. Hardware complexity for ABF configuration AESA Array of elements.

\begin{tabular}{|c|c|c|c|c|}
\hline & Cylindrical & $\begin{array}{c}\text { Planar } \\
\text { (same size) }\end{array}$ & $\begin{array}{c}\text { Planar } \\
\text { (same power) }\end{array}$ & $\begin{array}{c}\text { Rotating } \\
\text { (one AESA) }\end{array}$ \\
\hline Dimension & $4 \times 2 \mathrm{~m}$ & $4 \times 2 \mathrm{~m}$ & $3.14 \times 2 \mathrm{~m}$ & $4 \times 2 \mathrm{~m}$ \\
\hline K horizontal arrays & 54 & 54 & 54 & 54 \\
\hline $\begin{array}{l}\text { M elements in } \\
\text { horizontal array }\end{array}$ & 86 & 54 & 44 & 54 \\
\hline $\mathrm{B}$ receive beams & 12 & 12 & 12 & 12 \\
\hline Nh horns, TR, atten & $\begin{array}{c}\mathrm{K} \times \mathrm{M} \times 2= \\
9288\end{array}$ & $\begin{array}{c}\mathrm{K} \times \mathrm{M} \times 4= \\
11664\end{array}$ & $\begin{array}{c}\mathrm{K} \times \mathrm{M} \times 4= \\
9504\end{array}$ & $K \times M=2916$ \\
\hline Nph phase sifters & $\begin{array}{c}2(\mathrm{M} * \mathrm{~K} * \mathrm{~B})= \\
111456\end{array}$ & $\begin{array}{c}4\left(K^{*} \mathrm{M} * \mathrm{~B}\right)= \\
139968\end{array}$ & $\begin{array}{c}4(\mathrm{~K} * \mathrm{M} * \mathrm{~B})= \\
114048\end{array}$ & $\begin{array}{r}\mathrm{M} * \mathrm{~B} \times \mathrm{K} \\
34992\end{array}=$ \\
\hline Nsw switches & 9288 & 0 & 0 & 0 \\
\hline $\begin{array}{l}\text { Ncm combiners with } \\
\mathrm{N} \text { inputs }\end{array}$ & $\begin{array}{l}12 \text { with } 9288 \\
\text { input }\end{array}$ & $\begin{array}{c}12 \text { with } 11664 \\
\text { input }\end{array}$ & $\begin{array}{l}12 \text { with } 9504 \\
\text { input }\end{array}$ & $\begin{array}{l}12 \text { with } 2916 \\
\text { input }\end{array}$ \\
\hline $\begin{array}{l}\text { Nd dividers with B } \\
\text { outputs }\end{array}$ & $\begin{array}{c}9288 \text { with } 12 \\
\text { outs }\end{array}$ & $\begin{array}{l}11664 \text { with } 12 \\
\text { outs }\end{array}$ & $\begin{array}{l}9504 \text { with } 12 \\
\text { outs }\end{array}$ & $\begin{array}{l}2916 \text { with } 12 \\
\text { outs }\end{array}$ \\
\hline $\begin{array}{l}\text { Nck combiners with } \\
M^{*} \text { K outputs }\end{array}$ & 2 with 4644 outs & 4 with 2916 outs & 4 with 2376 outs & $\begin{array}{c}1 \text { with } 2916 \\
\text { outs }\end{array}$ \\
\hline
\end{tabular}

Table $\mathrm{T} 2$ shows the number of hardware elements under the same conditions as for the configuration for table T1. An incredibly large number of phase shifters is required, so the possibility of realizing such a configuration is very small. However, this configuration makes sense for small height radar at low frequencies with a small number of elements. For example, for a planar array with $\mathrm{M}=32$ 
elements, $\mathrm{K}=2$ horizontal rows with circular horns, $\mathrm{B}=4$ beams are sufficient. Then the number of required phase shifters for 4 grids is $4 * 32 * 2 * 4=1024$.

\subsection{Digital beam forming $\mathrm{DBF}$}

The $\mathrm{ABF}$ requires an unrealistically large number of phase shifters to configure the Array of elements. Also, the distribution network with dividers and combiners is very complex and spatially demanding. A solution could be sought in the Digital Beam Forming DBF. A simplified block diagram is shown in Fig. 20.

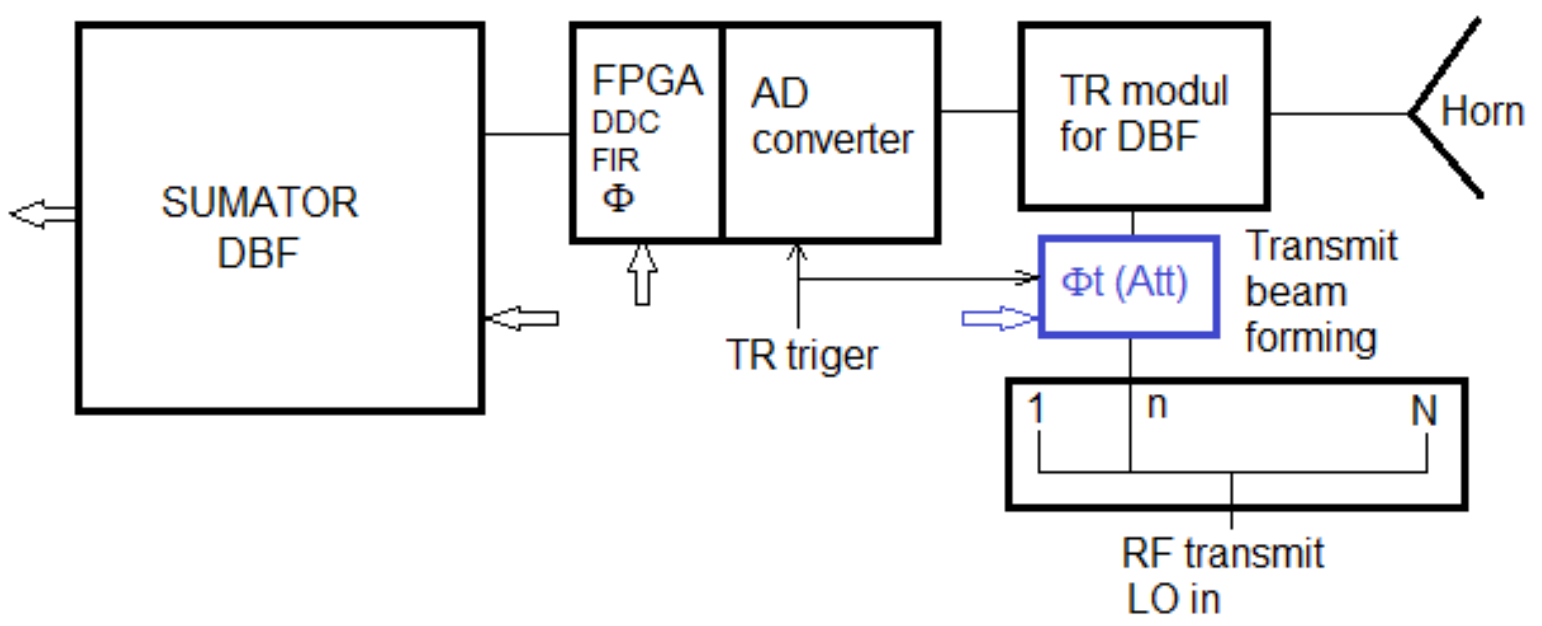

Fig. 20. Block schema DBF for Array of elements configuration.

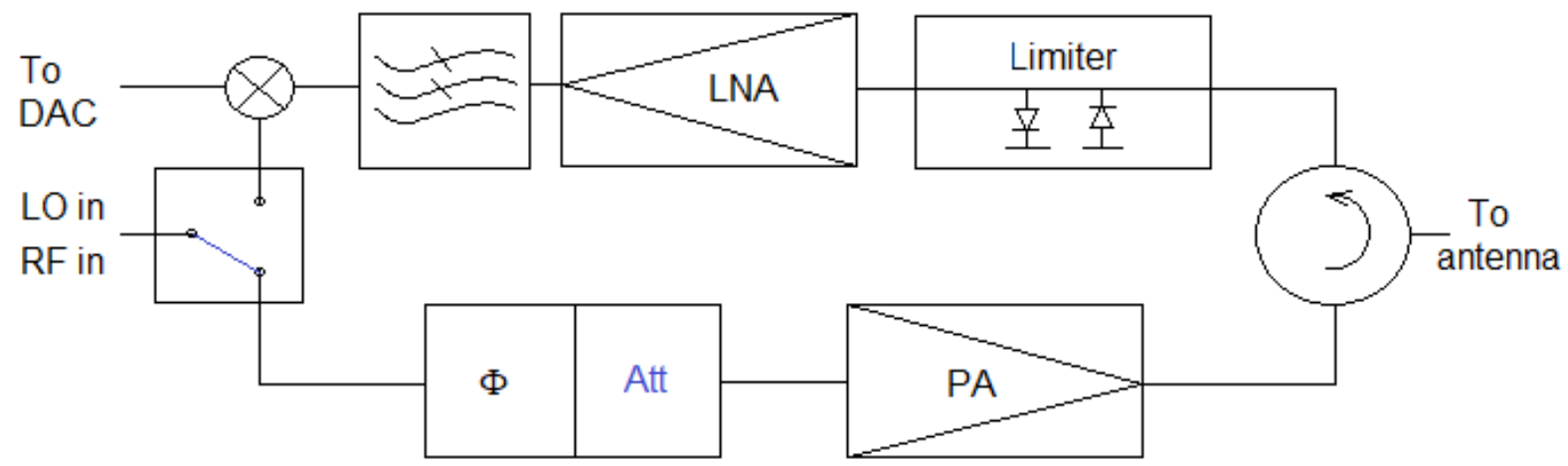

Fig. 21. TR module for Digital Beam Forming. 
The transmit side is the same as with ABF for Array of elements configuration. However, a different TR module is required, Fig. 21.

Separation of the transmitting and receiving part at the input must be by a PIN switch, not by a ferrite circulator. The $\Phi / A t t$ block is not required on the receiving side because the phase and amplitude distribution are done digitally in Sumator. The mixer is used for RF down conversion. It is useful, if possible, to have a symmetrical frequency suppression filter on the receiving side. Since the RF distribution network is uniform in amplitude and phase for the Tx signal, it is also suitable for LO signal distribution.

The signal from each TR module is digitized into an AD converter block. Digitization can be done at RF, IF or Base Band (BB) level. Digitization at the RF level is possible only for radars at very low frequencies. Also sampling on IF is possible only if IF is relatively low. The highest sampling frequency is limited by the maximum working frequency of the $\mathrm{AD}$ converter. In addition, there are problems associated with high frequency of clock for AD converters.

Distribution of high frequency clocks to each $\mathrm{AD}$ could be a problem. In order to avoid an error in the phase of the measured signal, the phase of the clock signal must be the same on all AD converters. This task could be solved by distributing the clock through the RF network for the distribution of Tx and LO signals. Assuming that the RF network is of the binary tree type the delay to each $\mathrm{AD}$ is the equal. Although the clock is pulse-shaped, the transmission in the BB is unsuitable, so a modulated RF carrier should be used. Suitable modulation could be BPSK. The circuit for digitization of the clock must be of good quality so that there is no phase error due to the unstable digitization threshold. For correct digitization, a signal for synchronization for all $\mathrm{AD}$ converters is needed.

A low IF value in not useful because it is difficult to solve the problem of symmetric frequency rejection for a wide RF band. On the other hand, the high value of IF brings the same problems that occur when sampling at the RF level. 
The most favourable solution seems to $\mathrm{BB}$, more precisely near to $\mathrm{BB}$. The price to be paid when converting to $\mathrm{BB}$ is twice noise level because there is no suppression of symmetrical frequency.

Regardless of whether the measurement is performed on RF, IF or BB, a relatively large gain of receiving signal is required. The noise from input of TR module must be amplified such to be at threshold level of $\mathrm{AD}$. The problem is to maintain the same phase and amplitude characteristic for each TR block. Although it is theoretically possible to calibrate and correct the errors in the receiving chain for each individual TR-AD chain, this is not recommended because it should be performed for each frequency channel.

Basic processing of the digitized signal (digital conversion, filtering, decimation) is performed in the FPGA for each TR module individually. The data rate of the processed signal can be low, even 5Msimpls/s. The processed signal is fed to the Sumator where the phase and amplitude adjustments, correction and summation are performed. The number of Sumators is equal to the number of receiving beams.

The RF part of the Beam Forming Network for DBF is quantitatively and qualitatively simple. However, digital transmission network can be very demanding. The number of digital channels for data transfer to the sumator could be of the order of 10000. Table T3 gives a quantitative view of the hardware for DBF.

The obvious problem is the organization of a digital data transfer network. It is necessary to transmit very large amount of data during one pulse. The physical number of entrances to the Sumator for a cylindrical antenna is of the order of 10000 , so the question of spatial arrangement arises. The situation can be improved by $\mathrm{K} / \mathrm{B}$ times if the summation is partly performed at the level of the vertical array. The data rate will be reduced by $\mathrm{K} /(2 \mathrm{~B})$ times.

A compromise solution is possible: $\mathrm{ABF}$ at the level of horizontal arrays, and DBF to form an array of horizontal arrays. However, this does not overcome problems with beam deformation as in the analogue Array of horizontal arrays configuration. 
Table T3. Configuration hardware complexity for DBF.

\begin{tabular}{|c|c|c|c|c|}
\hline & Cylindrical & $\begin{array}{c}\text { Planar } \\
\text { (same size) }\end{array}$ & $\begin{array}{c}\text { Planar } \\
\text { (same power) }\end{array}$ & $\begin{array}{c}\text { Rotating } \\
\text { (one AESA) }\end{array}$ \\
\hline Dimension & $4 \times 2 \mathrm{~m}$ & $4 \times 2 \mathrm{~m}$ & $3.14 \times 2 \mathrm{~m}$ & $4 \times 2 \mathrm{~m}$ \\
\hline K horizontal arrays & 54 & 54 & 54 & 54 \\
\hline $\begin{array}{l}\text { M elements in } \\
\text { horizontal array }\end{array}$ & 86 & 54 & 44 & 54 \\
\hline $\mathrm{B}$ receive beams & 12 & 12 & 12 & 12 \\
\hline Nh horns, TR, atten & $\begin{array}{c}\mathrm{K} \times \mathrm{M} \times 2= \\
9288\end{array}$ & $\begin{array}{c}\mathrm{K} \times \mathrm{M} \times 4= \\
11664\end{array}$ & $\begin{array}{c}\mathrm{K} \times \mathrm{M} \times 4= \\
9504\end{array}$ & $K \times M=2916$ \\
\hline Nadc AD converters & 9288 & 11664 & 9504 & 2916 \\
\hline Nfpga FPGA & 9288 & 11664 & 9504 & 2916 \\
\hline Nsum sumators DBF & $\begin{array}{l}12 \text { with } 9288 \\
\text { phase shifters }\end{array}$ & $\begin{array}{l}12 \text { with } 11664 \\
\text { phase shifters }\end{array}$ & $\begin{array}{l}12 \text { with } 9504 \\
\text { phase shifters }\end{array}$ & $\begin{array}{l}12 \text { with } 2916 \\
\text { phase shifters }\end{array}$ \\
\hline $\begin{array}{l}\text { Ndch total logical } \\
\text { channels inside } \\
\text { sumators }\end{array}$ & 111456 & 139968 & 114048 & 34992 \\
\hline $\begin{array}{l}\text { Ncabl total physical } \\
\text { data channels }\end{array}$ & 9288 & $4 \times 2916$ & $4 \times 2376$ & 2916 \\
\hline
\end{tabular}

\subsection{MBM for Array of vertical arrays}

Similar to horizontal arrays, an Array of vertical arrays configuration can be formed on the receiving side. When it comes to a planar array, it is logical to expect the same problems with beam deformation to occur as in an Array of horizontal arrays configuration. On Fig. 22. is shown an example of drastic deformation of the pattern for a flat AESA.

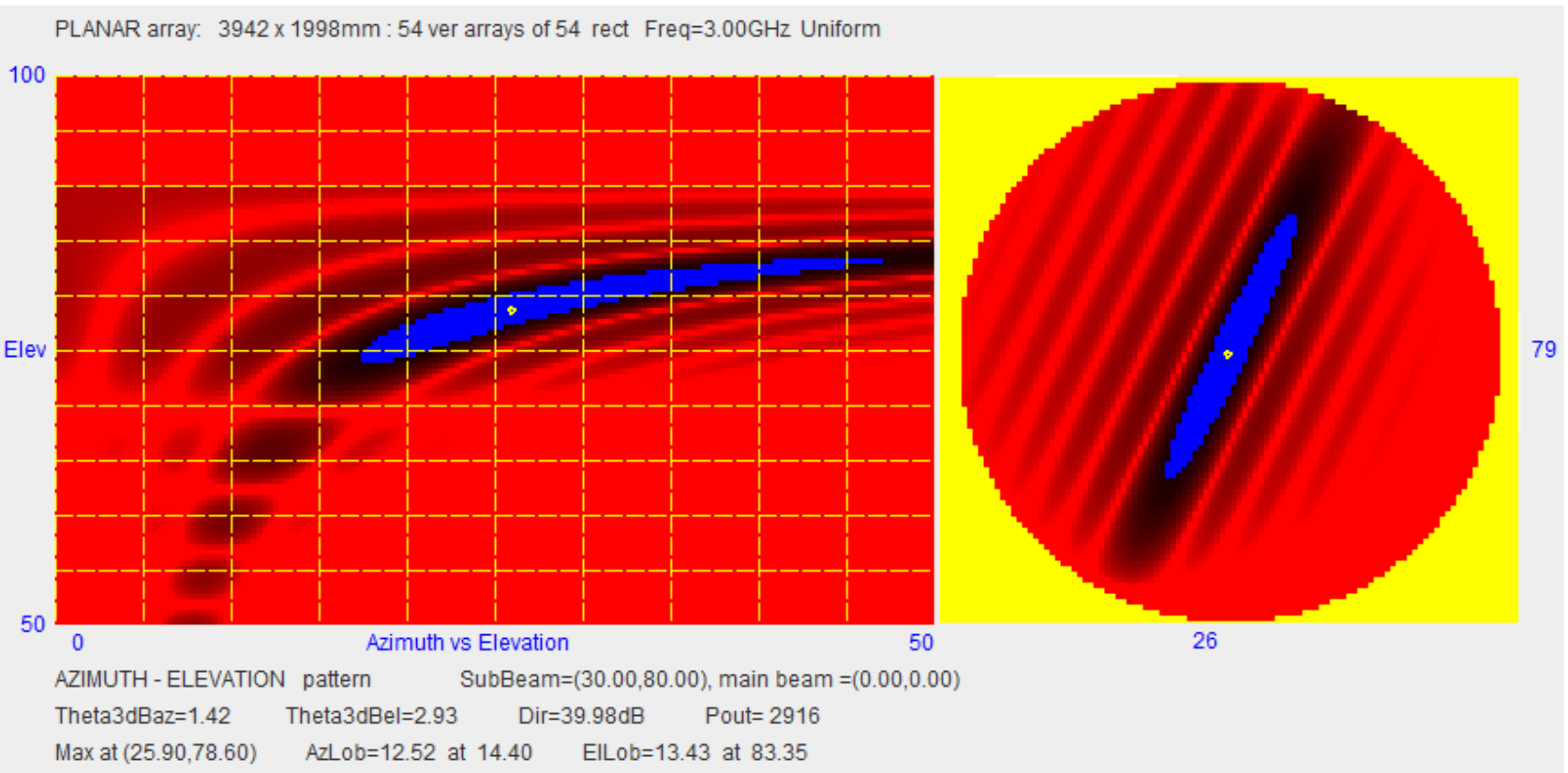

Fig. 22. Very visible pattern distortion of planar array of vertical arrays. 


\subsection{Special vertical arrays configuration for Very fast scanning in near zone}

\section{VFSNZ}

It could be expected no significant beam deformation for cylindrical array during the sub-beam forming in horizontal plane. This is because the geometry of the cylindrical array is invariant in azimuth.

In order to be able to use vertical arrays configuration, the main Tx beam must be spread in azimuth. However, this would not lead to faster scanning in the far zone as the gain of antenna in transmit mode would decrease.

However, a special variant of array of vertical arrays significantly speeds up scanning in the near zone. In this configuration, all vertical arrays are the same. In the transmission mode, they are all equally excited and steered to the same elevation angle $\beta$. Due to the circular symmetry of the cylindrical antenna, the azimuth pattern of the antenna is practically uniform in the entire $360^{\circ}$ range.

In the receiving mode, the required number of sub-beams is formed to cover the complete $360^{\circ}$ azimuth. Let's say that the horizontal sub-beams are spaced by a beam width of $3 \mathrm{~dB}$, and that the beam width is $3^{\circ}$. In that case, 120 horizontal receiving sub-beams are needed to cover the complete azimuth. Only one burst is enough to scan the complete azimuth at $\beta$ elevation.

The scanning speed of the complete space depends on the beam width in the vertical plane. If the average beam width in vertical plane is $6^{\circ}$, then only 15 bursts are needed to scan the entire space in the near zone. If pulse repetition time is about $1 \mathrm{~ms}$, and burst is composed of two pulses, then the scan time is only about $30 \mathrm{~ms}$.

The limits of the near zone are determined by the range for the given probability of detection and false alarm. If all operating conditions are the same as in long range mode, then the range decreases by the fourth root of the decreases of antenna gain in transmit mode. If, for example, the gain is reduced 100 times, then the range drops to about $30 \%$ of the range for the far zone. Minimum range is determined by pulse width.

The question of beam deformation in the VFSNZ configuration is raised. The shape of the main beam for different elevation angles $\beta$ is shown on the Fig. 23. The 
upper part displays receive beam shape at conical section with constant elevation. When elevation increases, the beam spreads. A smaller number of receive sub beams is required for the entire azimuth of $360^{\circ}$. The pattern on horizontal plane is shown at lower part of Fig. 23. The beam width is almost constant.

CILINDRICAL : $3919 \times 1964 \mathrm{~mm}: 76$ ver arrays of 28 round Freq $=3.00 \mathrm{GHz}$ Uniform
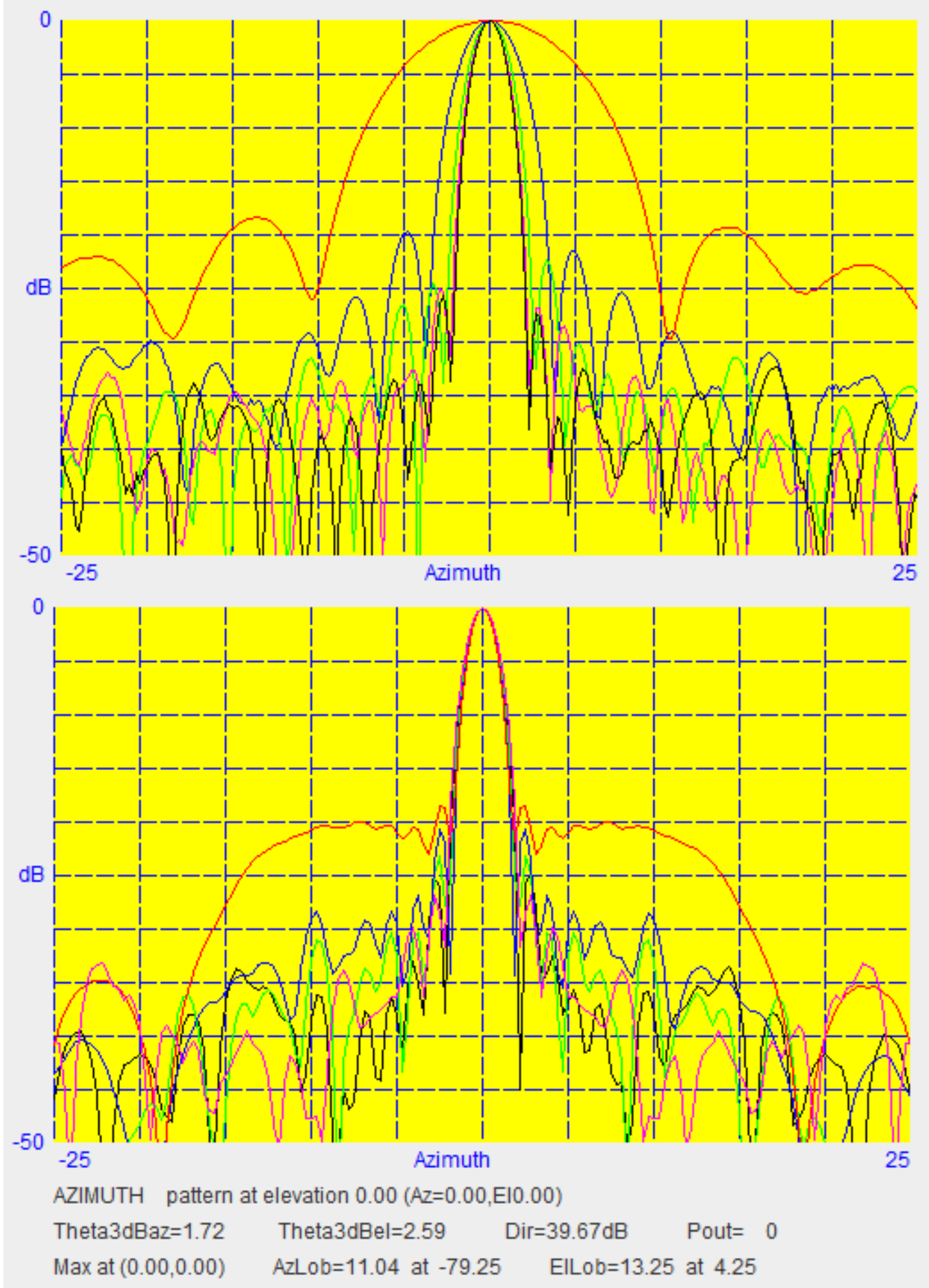

Fig.23. Main beam spreading in azimuth of Very fast scanning cylindrical array: red -80 , blue -60 , green -40 , magenta -20 , black -0 . 
It can be seen that pattern distortions are almost insignificant for an angle up to $60^{\circ}$, Fig. 24. The distortions at $80^{\circ}$ are significant, but the system is still usable.

For complete surveillance, the standard far-zone surveillance mode can be combined with the VFSNZ near-zone mode.

CILINDRICAL : $3919 \times 1964 \mathrm{~mm}: 76$ ver arrays of 28 round Freq $=3.00 \mathrm{GHz}$ Uniform
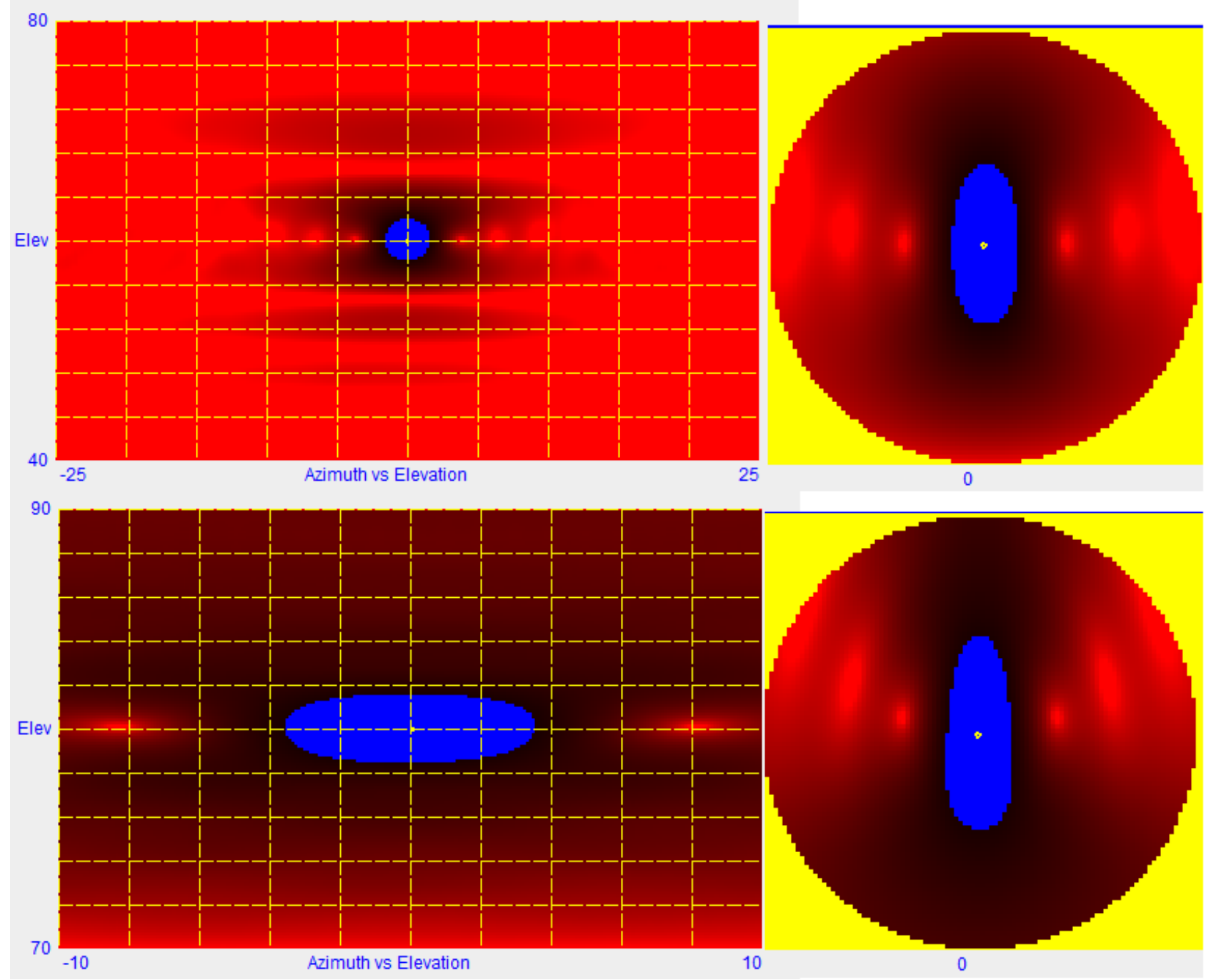

Fig. 24. Azimuth pattern of Very fast scanning cylindrical array VFSNZ at elevation $60^{\circ}$ (up) and $80^{\circ}$ (down).

The construction of VFSNZ could look complicated. But by using a combined $\mathrm{ABF}$ and DBF beam forming, the construction becomes feasible. The formation of the sub-beams is done in the following steps:

1. The analogue summation of the element's signals is performed at the RF level. 
2. The summed signals are converted to a frequency suitable for DA conversion. It can be near to BB, or IF frequency.

3. Sampling with DA converters and processing is performed. The signal with a relatively low data rate is now ready for digital beam forming DBF.

4. DBF for the required number of sub-beams is performed in digital sumators.

The question of the feasibility of a digital data transfer network in VFSNZ is raised. The logical block diagram of the data distribution network for VFSNZ is shown in Fig.25.

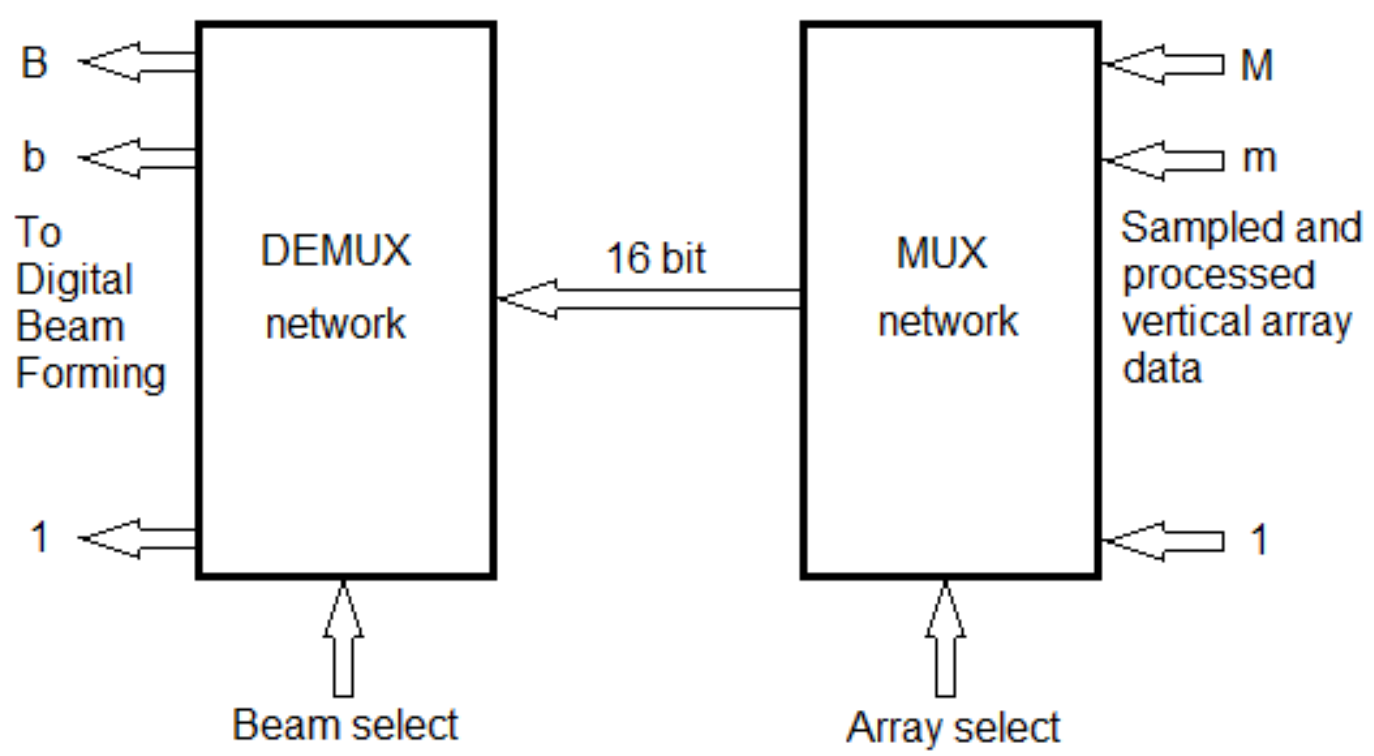

Fig. 25. Data transmission network for Very fast scanning in near zone.

The number of vertical $\mathrm{M}$ arrays is equal to the number of elements in the horizontal circular array. A multiplexer is needed for transfer the data of $\mathrm{m}$ vertical arrays, one by one, to the demultiplexer. Assume that 16-bit parallel transmission is sufficient to transmit a single (decimated) data. If the data rate of the decimated data is $5 \mathrm{MHz}$, and the receiving time is $1 \mathrm{~ms}$, the required number of data from one array is 5000. For a cylindrical AESA with $M=200$ vertical arrays, the total number of data to be transmitted for one pulse is $1,000,000$. A $1 \mathrm{Gbit} / \mathrm{s}$ transmission clock is required. In practical implementation, by optimization and parallelization, the clock can be further reduced. 
Based on this analysis, it is concluded that a VFSNZ can be realized. Of course, it is necessary to provide an adequate system for target detection and tracking, which will be able to process signals from all sub-beams.

\section{Range}

\subsection{Range in far zone}

The radar range is proportional

$$
\mathrm{R}^{4} \sim\left(\mathrm{P}_{\mathrm{rad}} * \mathrm{G}_{\mathrm{t}} * \mathrm{G}_{\mathrm{r}} * \mathrm{~S} * \mathrm{n}\right) /\left(\mathrm{SN}_{\min }\right)
$$

where

$\mathrm{P}_{\mathrm{rad}}$ - radiated power

$\mathrm{G}_{\mathrm{t}}$ - antenna gain in transmit mode

$\mathrm{G}_{\mathrm{r}}$ - antenna gain in receive mode

$\mathrm{S}$ - scattering coefficient, of the target

$\mathrm{n}$ - number of pulses in burst

$\mathrm{SN}_{\text {min }}$ - minimal signal to noise ration for requested probability of target detection and false alarm.

The radiated power is

$$
\mathrm{P}_{\mathrm{rad}}=\Sigma \mathrm{P}_{\mathrm{i}}-\mathrm{L} \quad \mathrm{i}=1: \mathrm{N}
$$

where the $\mathrm{N}$ is the number of radiating elements, $\mathrm{P}_{\mathrm{i}}$ is power of single element, $\mathrm{L}$ losses on cables and attenuators. The antenna gain is roundly proportional to the beam width

$$
\mathrm{G}_{\mathrm{t}} \sim \theta_{3 \mathrm{dbvt}} * \theta_{3 \mathrm{dbht}}
$$

where $\theta_{3 \mathrm{dbvt}}$ is width of transmit beam in vertical plane, $\theta_{3 \mathrm{dbht}}$ is width of transmit beam in horizontal plane

$$
\mathrm{G}_{\mathrm{r}} \sim \theta_{3 \mathrm{dbvr}} * \theta_{3 \mathrm{dbhr}}
$$

where $\theta_{3 \mathrm{dbvr}}$ is width of receive beam in vertical plane, $\theta_{3 \mathrm{dbhr}}$ is width of receive beam in horizontal plane.

The equation (13) is valid if the integrator is ideal and the efficiency of the integrator does not depend on the number of pulses $n$. 
It is of interest to determine the relative range of radar with a planar AESA in relation to radar with a cylindrical AESA at the same scanning speed in azimuth, which means with the same number of pulses $n$ in the burst. The comparison is made under the conditions:

- S coefficient of the target is the same,

- $\mathrm{SN}_{\text {min }}$ minimum required signal to noise ratio is the same,

- transmit and receive beam is directed at the same elevation angle.

Then, for both types of radar, the range in the direction of the angle $\alpha$ in azimuth depends only on the radiated power and the width of transmit and receive beam:

$$
\mathrm{R}(\alpha)^{4} \sim \mathrm{P}_{\mathrm{rad}} /\left(\theta_{3 \mathrm{dbvt}}(\alpha) * \theta_{3 \mathrm{dbht}}(\alpha) * \theta_{3 \mathrm{dbvr}}(\alpha) * \theta_{3 \mathrm{dbhr}}(\alpha)\right)
$$

For a cylindrical antenna, the beam width in azimuth is constant, so the range does not depend on the angle $\alpha$. The same is for the rotating radar with a planar AESA.

\subsection{Surveillance time}

During the comparison of the azimuth scan speed, a constant elevation angle with the maximum range is taken. It is assumed that the number of pulses in the burst of the cylindrical antenna array is 10 . It is of interest to determine the range in the far zone, where a larger number of pulses is needed to achieve the given range. It assumes that the level of clutter and interferences is evenly distributed over the azimuth.

It is assumed that the azimuth scanning is performed in discrete directions. The angles of the observing directions are spaced half the beam 3dB-width in the azimuth plane.

With a cylindrical antenna, the beam angle is fixed during scanning, so the number of pulses in the burst $\mathrm{n}_{\mathrm{c}}$ is also constant and proportional to:

$$
\mathrm{n}_{\mathrm{c}} \sim\left(\theta_{\mathrm{htc}} * \theta_{\mathrm{vtc}} * \theta_{\mathrm{hrc}} * \theta_{\mathrm{vrc}}\right) / \mathrm{P}_{\mathrm{radc}}
$$

where

$$
\begin{aligned}
& \mathrm{P}_{\mathrm{radc}}-\text { radiated power of radar with cylindrical AESA, } \\
& \theta_{\mathrm{htc}}-\text { beam width in the horizontal plane in transmit mode, } \\
& \theta_{\mathrm{vtc}}-\text { beam width in the vertical plane in transmit mode, }
\end{aligned}
$$


$\theta_{\text {hrc }}$ - beam width in the horizontal plane in receive mode,

$\theta_{\text {vrc }}-$ beam width in the vertical plane in receive mode.

The time required to scan a sector of $180^{\circ}$ is:

$$
\mathrm{T}_{\mathrm{c}}=2 \mathrm{n}_{\mathrm{c}} \mathrm{N}_{\mathrm{c}}
$$

where $\mathrm{N}_{\mathrm{c}}$ is the number of discrete observation directions in the sector $0^{\circ}$ to $90^{\circ}$ :

$$
\mathrm{N}_{\mathrm{c}}=90 /\left(\theta_{\mathrm{h}} / 2\right)
$$

where $\theta_{\mathrm{h}}$ is smaller one between $\theta_{\mathrm{hrc}}$ and $\theta_{\mathrm{htc}}$.

$\mathrm{T}_{\mathrm{c}}$ is also the scanning time of the entire azimuth of $380^{\circ}$, because the other half of the cylindrical antenna scans other half of the azimuth.

The beam width of planar AESA increases with the change of the observation angle $\alpha$ from $0^{\circ}$ to $45^{\circ}$. Therefore, the directions of observation are more spaced as the direction changes towards $45^{\circ}$. As the beam width increases, the number of pulses required in the burst to keep the range constant also increases. The number of pulses in the burst $\mathrm{n}_{\mathrm{p}}$ is proportional

$$
\mathrm{n}_{\mathrm{p}}(\alpha) \sim\left(\theta_{\mathrm{htp}}(\alpha) * \theta_{\mathrm{vtp}}(\alpha) * \theta_{\mathrm{hrp}}(\alpha) * \theta_{\mathrm{vrp}}(\alpha)\right) / \mathrm{P}_{\mathrm{radp}}
$$

where

$\mathrm{P}_{\text {radp }}$ - radiated power of radar with planar AESA,

$\theta_{\text {htp }}$ - beam width of planar AESA in the horizontal plane in transmit mode,

$\theta_{v t p}$ - beam width of planar AESA in the vertical plane in transmit mode,

$\theta_{\text {hrp }}$ - beam width of planar AESA in the horizontal plane in receive mode,

$\theta_{\text {vrp }}$ - beam width of planar AESA in the vertical plane in receive mode.

The time required to scan the $90^{\circ}$ sector is

$$
\mathrm{T}_{\mathrm{p}}=2 \Sigma \mathrm{n}_{\mathrm{pi}} \quad \mathrm{i}=1: \mathrm{N}_{\mathrm{p}}
$$

where $\mathrm{N}_{\mathrm{p}}$ is the required number of positions to overwrite the angle from $0^{\circ}$ to $45^{\circ}$. $\mathrm{Tp}$ is also the time of scanning the whole azimuth, since 4 arrays are used at the same time. It is assumed sign-to-noise ratio in the receiver is proportional to the number of pulses in the burst. This is true only in the case of an ideal integrator. Otherwise, the contribution of $n_{p}$ pulses is smaller, so the scan time calculated in (22) is the shortest 
possible.

In radar with one rotating planar AESA, the beam width is constant during scanning, so the number of pulses in the burst $\mathrm{n}_{\mathrm{r}}$ is also constant. It is assumed that the number of pulses in the burst $n_{r}$ is equal to $n_{c}$ for the same range as the radar with a cylindrical AESA. If the azimuth beamwidth in receive mode is less than or equal to the azimuth beamwidth in transmit mode, the relative azimuth scanning time for a rotating AESA radar relative to a cylindrical AESA radar is

$\mathrm{T}_{\mathrm{r}}=2 \mathrm{~T}_{\mathrm{c}}\left(\theta_{\mathrm{htr}} * \theta_{\mathrm{vtr}} * \theta_{\mathrm{vrr}} * \mathrm{P}_{\mathrm{radc}}\right) /\left(\theta_{\mathrm{htc}} * \theta_{\mathrm{vtc}} * \theta_{\mathrm{vrc}} * \mathrm{P}_{\mathrm{radr}}\right)$

where

$\mathrm{P}_{\mathrm{radr}}-$ radiated power of radar with rotating planar AESA,

$\theta_{\text {htr }}$ - beam width of rotating AESA in the horizontal plane in transmit mode,

$\theta_{\mathrm{vtr}}$ - beam width of rotating AESA in the vertical plane in transmit mode,

$\theta_{\mathrm{vrr}}$ - beam width of rotating AESA in the horizontal plane in receive mode.

The beam width in (23) is the same as for the planar AESA for $0^{\circ}$ azimuth.

Of course, the azimuth scanning speed of rotating radar could be reduced 2 or 4 times by adding 1 or 3 additional rotating AESA.

It is shown, on Fig. 26. the graph of the range (red line) of planar AESA normalized to the range of cylindrical AESA, as a function of azimuth. The range is calculated for the case with the same number of pulses in the burst. On the picture above, the number of TR modules is same for both cylindrical and planar AESA. The range of planar AESA is smaller, especially at angles for from the normal.

The figure below shows the range of a planar AESA with the same size, and thus with higher RF power. Even then, the range of the cylindrical AESA is slightly higher, because the radiated power is higher. The cylindrical antenna in the transmitting mode radiates with half of the installed RF power for one beam. If some of TR modules of cilindrical AESA were switched off, the range would be smaller then the range of planar one at azimuth of $0^{\circ}$.

The scanning time of a certain direction as a function of azimuth is shown by blue line. The line is stepped because the directions of observation are discrete. It can be seen that the observation time in directions far from $0^{\circ}$ increases because the gain 
of the antenna decreases.

CILINDRICAL : $3139 \times 1998 \mathrm{~mm}$ : $44 \times 54$ elements rect Freq $=3.00 \mathrm{GHz}$ Uniform

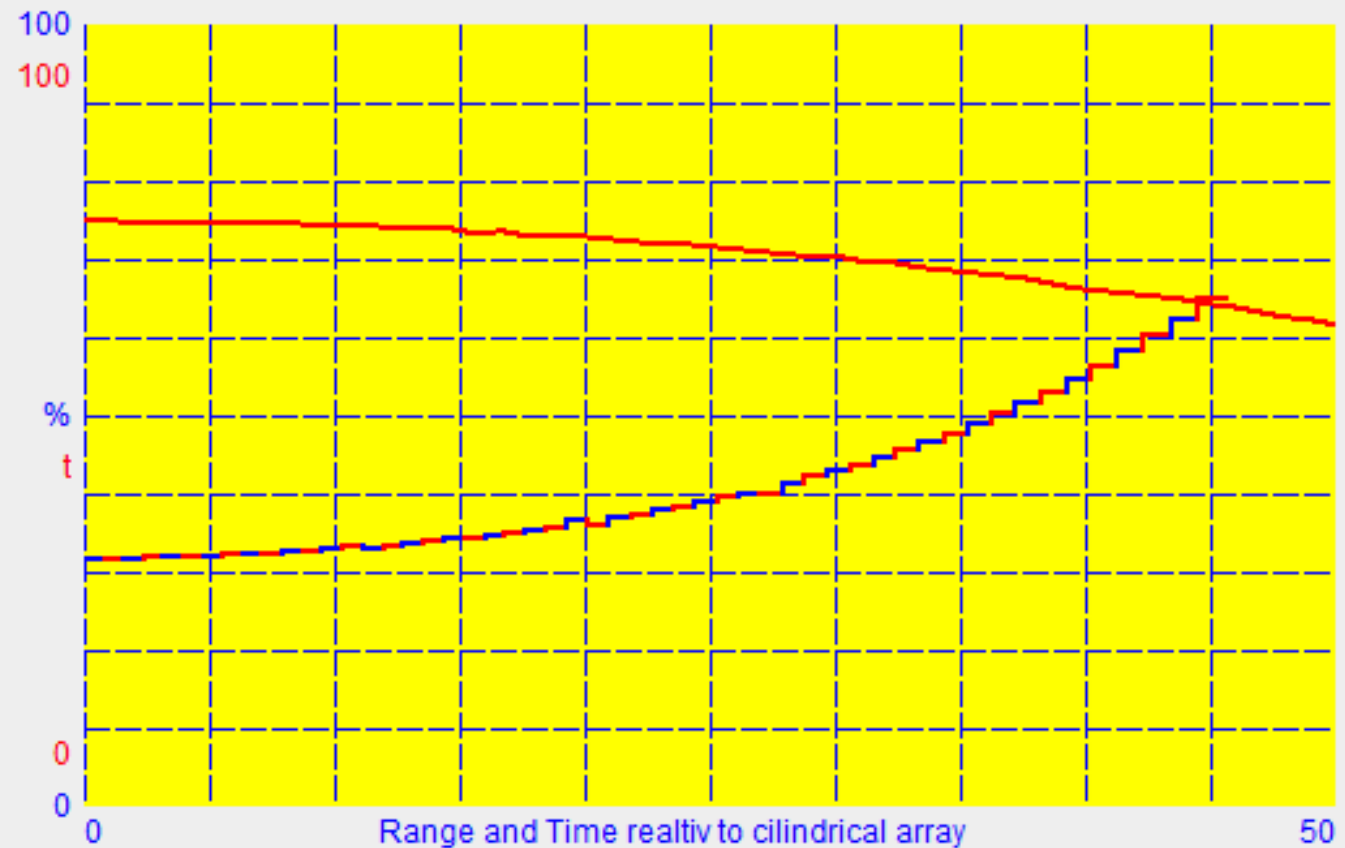

Planar array: RANGE and Scanning TIME relative to Cilindrical, Pplanar $=2376$

Theta3dBaz=1.24 Theta3dBel $=2.54 \quad$ Dir $=41.18 \mathrm{~dB} \quad$ Pout $=4644$

Scaning Time: Cilindrical: 0.31 Planar: 0.45

CILINDRICAL : $3942 \times 1998 \mathrm{~mm}$ : $54 \times 54$ elements rect Freq=3.00GHz Uniform

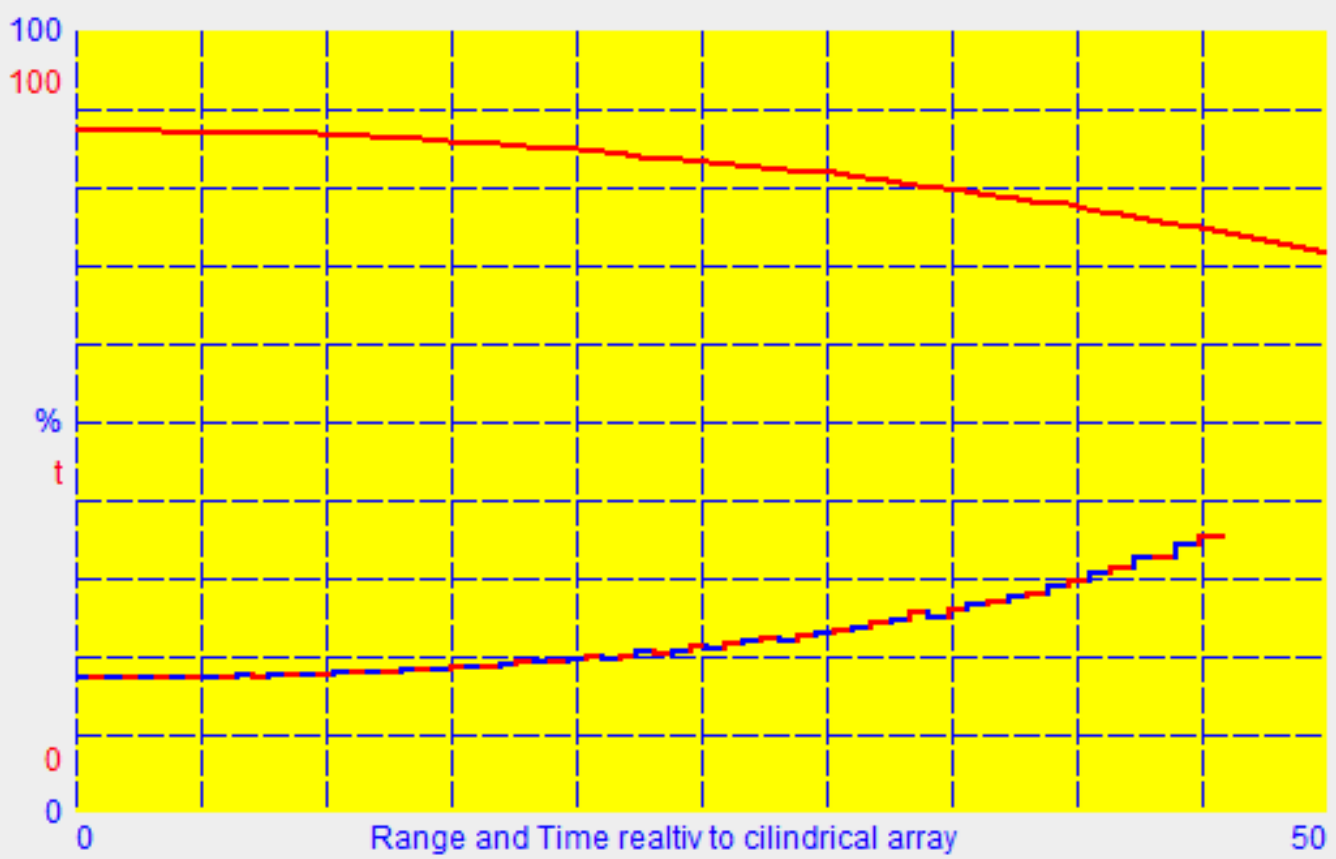

Planar array: RANGE and Scanning TIME relative to Cilindrical, Pplanar $=2916$

Theta3dBaz=1.24 Theta3dBel=2.54 Dir $=41.18 \mathrm{~dB} \quad$ Pout $=4644$

Scaning Time: Cilindrical: 0.31 Planar: 0.30

Fig. 26. Range (red) and Scanning time (blue) of planar AESA with same installed RF power (up) and same size (down) normalized to cylindrical AESA. 
The bottom text line on Fig. 26. shows the total scan time of the complete azimuth of $360^{\circ}$. The result confirms the expectation of a similar scan time for same size antennas. Radar scanning time with one rotating planar AESA is roundly 4 times longer than one with a prismatic antenna.

\subsection{Range in vertical plane}

It is interesting to calculate the radar range in the vertical plane in MBM mode. It is assumed that the main transmission beam is directed to the elevation for which the maximum range is desired. The main receiving sub-beam is directed in the same direction. Other receiving sub-beams are deflected for a certain angle in the vertical plane, for example for a beam width of $1 \mathrm{~dB}$. The normalized range is calculated relative to the maximum range.

CILINDRICAL : $3919 \times 982 \mathrm{~mm}: 14$ hor arrays of 76 round Freq $=3.00 \mathrm{GHz}$ Uniform

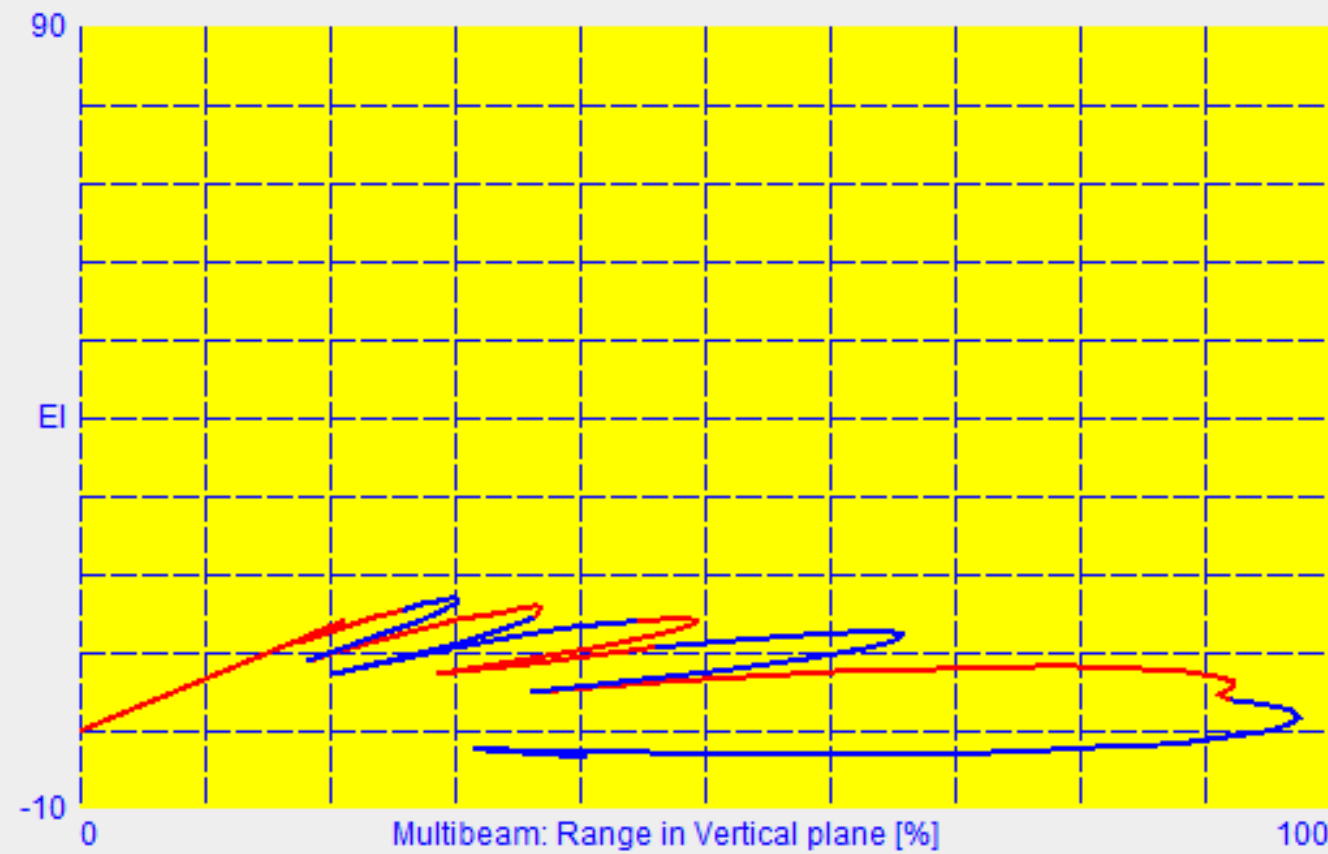

RANGE in vertical plane normalized to main beam: $(0.00,2.00)$

Theta3dBaz=1.49 Theta3dBel=5.17 Dir $=73.19 \mathrm{~dB} \quad$ Pout $=1064$

From -5 to 35 , Number of beams 9

Fig. 27. Range in vertical plane of Cylindrical AESA with uniform amplitude distribution.

In order to achieve the longest possible range and reduce the scanning time, it is necessary to radiate as much power as possible. Maximum radiated power is 
achieved with a uniform amplitude distribution. Figures 27, 28 and 29 show the range in the vertical plane with a uniform amplitude distribution. It can be seen from the figures, that generally, it is possible to perform scanning in the vertical plane with a uniform amplitude distribution on the transmission. This is especially pronounced with AESA of low height $\mathrm{H}$.

In the diagrams, the analysis of the range in the vertical plane is done for elevation up to $30^{\circ}$. At higher elevation angles, the dominant problem is beam distortion, not the strength of the receiving signal. High elevations can be covered by some other means such as additional AESA, additional horizontal array directed upwards, by conical or pyramidal AESA etc. But this is no longer a cylindrical or a planar AESA, so it is not analysed in this paper.

PLANAR array: $3888 \times 982 \mathrm{~mm}: 14$ hor arrays of 48 round Freq $=3.00 \mathrm{GHz}$ Uniform

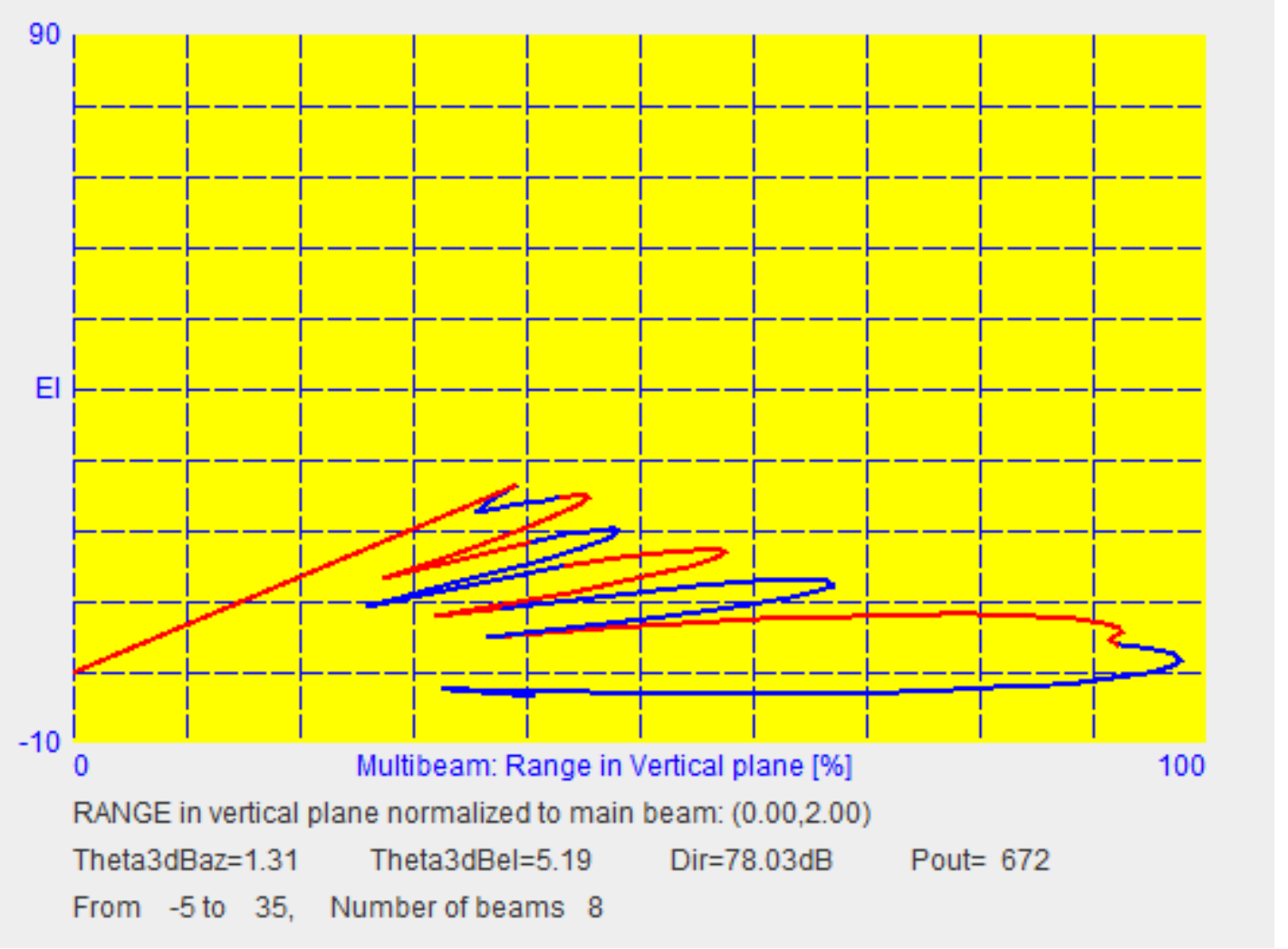

Fig. 28. Range in vertical plane of Planar AESA with uniform amplitude distribution at azimuth $0^{\circ}$. 
PLANAR array: $3888 \times 982 \mathrm{~mm}: 14$ hor arrays of 48 round Freq $=3.00 \mathrm{GHz}$ Uniform

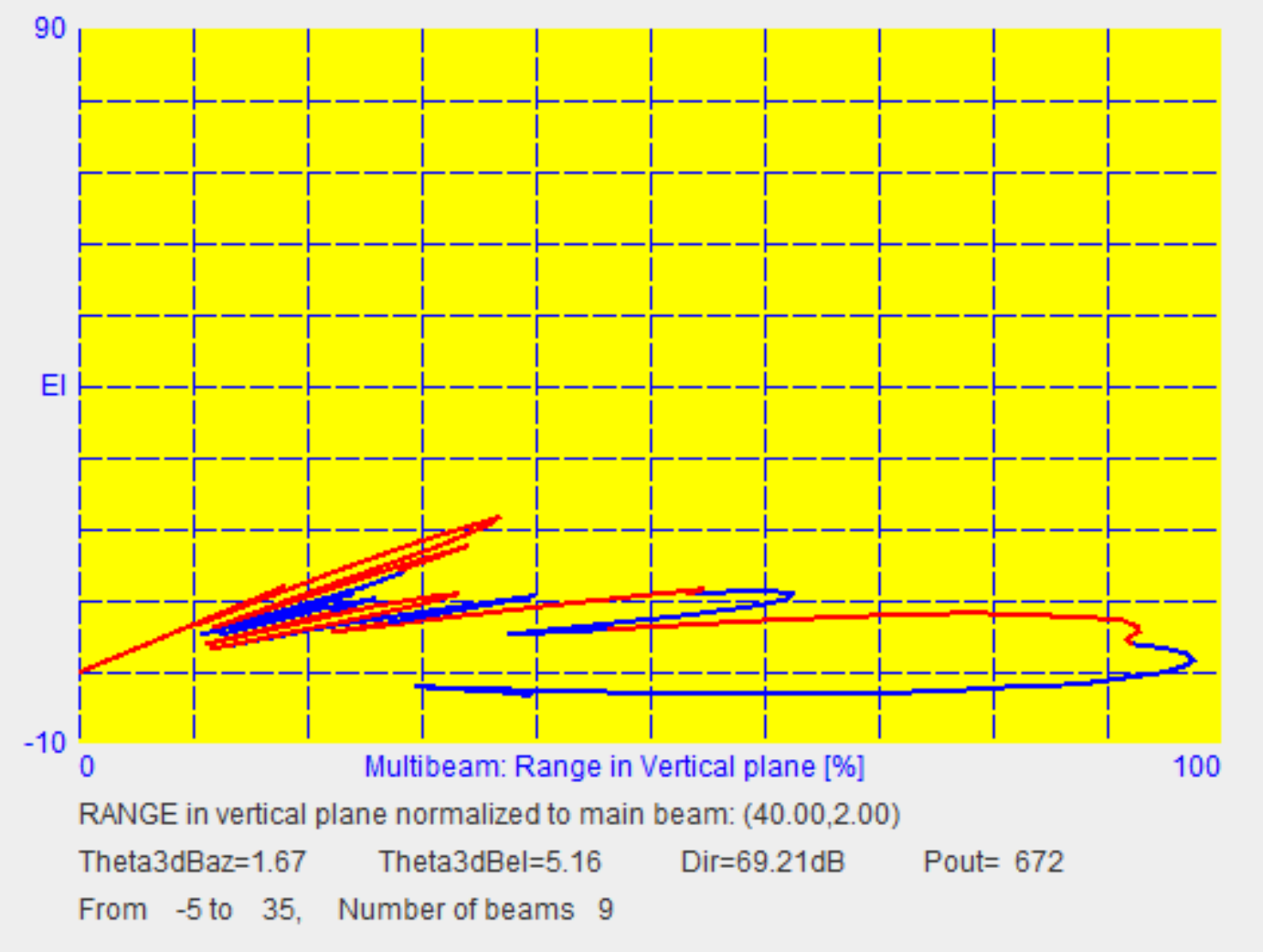

Fig. 29. Range in vertical plane of Planar AESA with uniform amplitude distribution at azimuth $40^{\circ}$.

\section{Tolerances}

AESA for surveillance radar is a system with a large number of components. In order to obtain the correct shape of the pattern, it is necessary that the electrical characteristics and position of the component in space be very precise. The question of tolerance arises when developing AESA. The question of the tolerance of the number of defective components during operation also arises. It should be specified at what number of defective components the system should be switched off.

In order to be able to specify the accuracy in the fabrication of electromechanical components, the maximum allowable error in the phase and amplitude distribution must be determined. The Random function in the program is used for this. For each radiating element, the value of phase and amplitude is set in the vicinity of the calculated value within the prescribed maximum error. The defective elements are chosen randomly. Their amplitude is set to 0 . 
PLANAR array: $3942 \times 1998 \mathrm{~mm}$ : $54 \times 54$ elements rect Freq $=3.00 \mathrm{GHz}$ Optimized

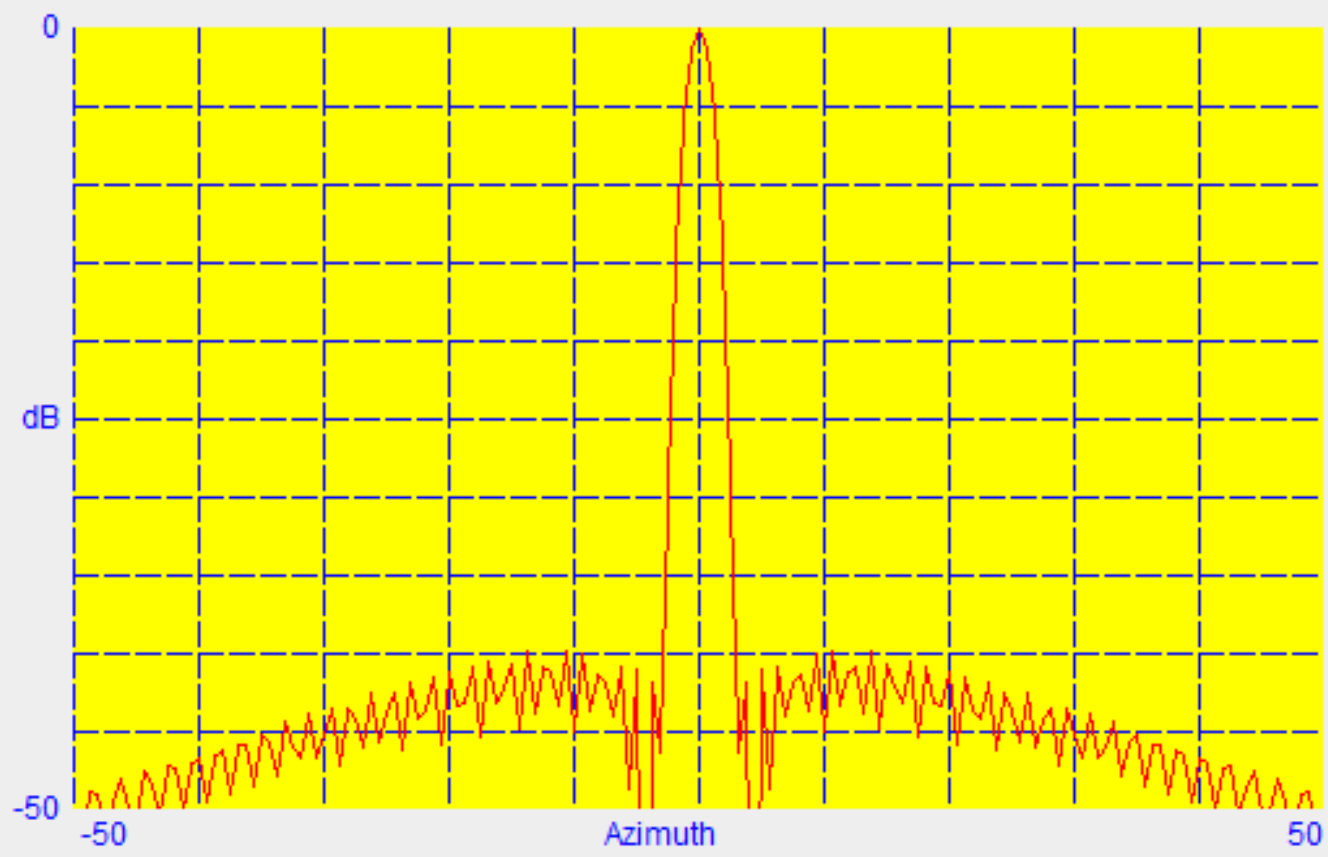

AZIMUTH pattern at elevation 0.00

Spot $=(0.00,0.00)$

Theta $3 \mathrm{dBaz}=1.84$ Theta $3 \mathrm{dBel}=2.54 \quad \mathrm{Dir}=39.46 \mathrm{~dB} \quad$ Pout $=1609$

Max at $(0.00,0.00) \quad$ AzLob $=39.65$ at $10.75 \quad$ ElLob $=13.32$ at 4.00

PLANAR array: $3942 \times 1998 \mathrm{~mm}$ : $54 \times 54$ elements rect Freq $=3.00 \mathrm{GHz}$ Optimized

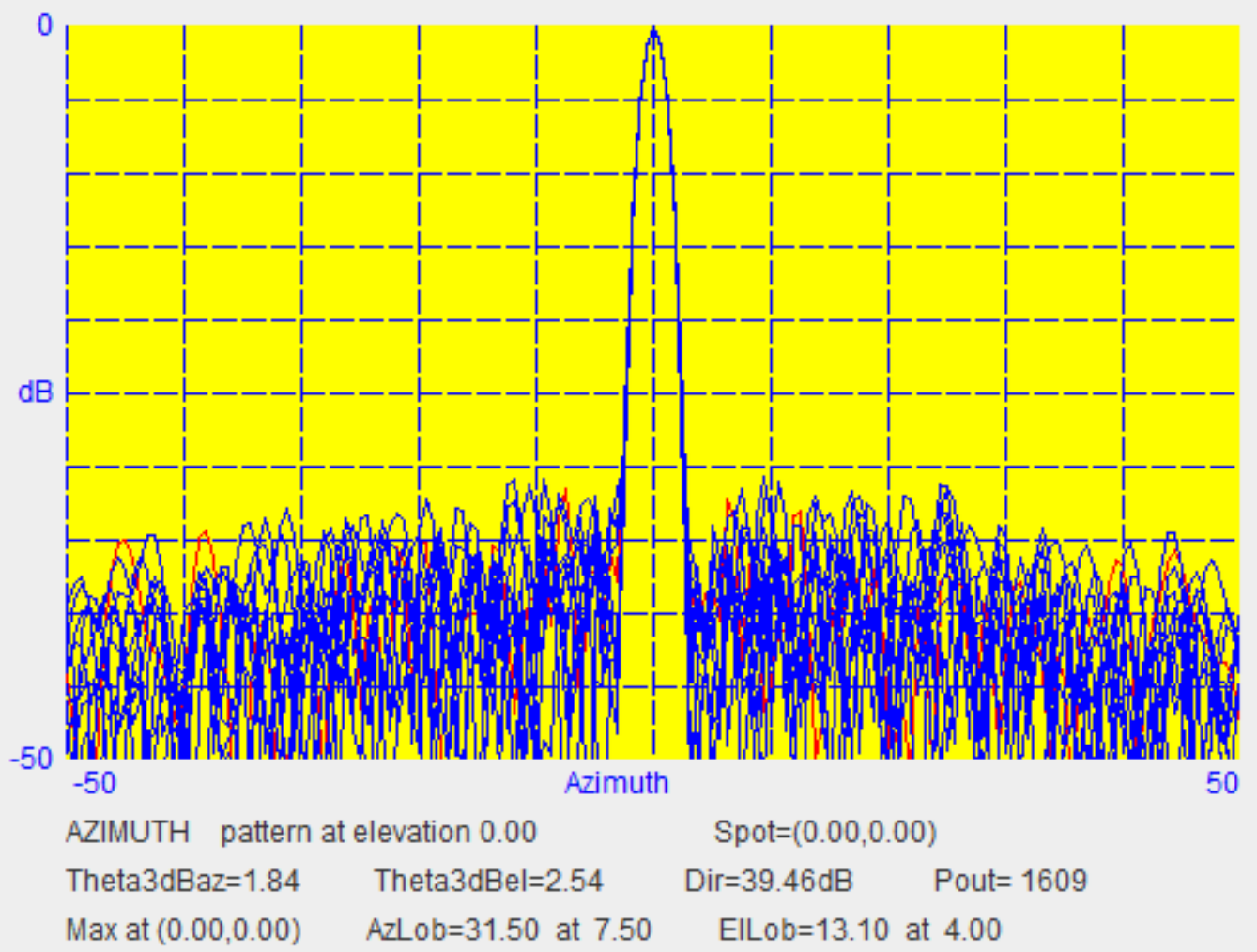

Fig. 30. Random analysis. 
The program in Random function calculates the pattern and allow monitoring of the degradation of the characteristic of interest. On Fig. 30. is shown the example of the effect of errors to the level of lateral lobes for planar AESA with optimized distribution. The upper graph has a pattern with accurate data, and the lower one has errors. The phase error is randomly distributed from $0^{\circ}$ to $90^{\circ}$ to all radiated elements. The amplitude error is from $0 \mathrm{~dB}$ to $2 \mathrm{~dB}$. The number of faulty elements is 20.

Based on the acceptable maximum error, the electrical and mechanical characteristics of the components can be specified. Faults affected by all components in the chain from the Tx input to the beam forming output Rx should be taken in account.

The error in the spatial position directly affects the phase characteristic. The mechanical construction must be such that the position errors are within the specified limits. It is especially difficult to organize a cylindrical AESA. An accuracy of a few $\mathrm{mm}$ must be provided in an entire cylinder with a diameter of $4 \mathrm{~m}$.

\section{Transportability}

Transportability is an important tactical feature of radar. Particularly significant is the case when the radar is wider than $2.45 \mathrm{~m}$ standard trucks or has to be disassembled for some other reason.

The cylindrical antenna can be made to be detachable, but the assembling is complicated, among other things, due to the offset arrangement of radiating elements. It is desirable that the cylindrical AESA does not disassemble. If it disassembles, it is necessary to provide such a construction that will provide assembling with the required precision of only a few millimetres. In general, the construction of a cylindrical structure is more complex than flat ones.

Radar with mechanical rotation of planar AESA usually has to be levelled before start.

The most suitable variant for disassembly is radar with prismatic AESA. Assembly after transport is not critical, because the prismatic antenna system essentially behaves like a system of 4 independent coordinated AESA. The distance 
between 4 AESA is not strictly defined. This gives an additional tactical advantage to this system. Radar can be installed in places where it is difficult to provide visibility without obstacles in all directions. For example, it can be installed on 4 sides of a tall building, not necessarily on top. It also does not have to be at the top of the hill, it can be installed on the edges. The situation is the same with the installation on the ship or aircraft.

Prismatic radar has another tactical advantage: in a situation where all 4 observation sectors are not in the interest, the unnecessary flat AESA can be simply omitted.

Big stationary low-frequency radars for a very large range must be transported in a disassembled state. In this case, the variant with a cylindrical antenna of small height is the most suitable.

\section{Conclusions}

T4. Tactical characteristics summary.

\begin{tabular}{|l|c|c|c|}
\hline \multicolumn{1}{|c|}{ Tactical characteristics } & $\begin{array}{c}\text { Mechanically } \\
\text { rotated } \\
\text { planar AESA }\end{array}$ & $\begin{array}{c}\text { Prismatic } \\
\text { system } \\
\text { of 4 } \\
\text { planar } \\
\text { AESA }\end{array}$ & $\begin{array}{c}\text { Cylindrical } \\
\text { AESA }\end{array}$ \\
\hline Multi mode & NO & Yes & Yes \\
\hline Skipping non active sectors & No & Yes & Yes \\
\hline Adapting rotation speed & Yes & No & Yes \\
\hline Constant pattern shape & Small & Hight & Hight \\
\hline Radiated power possibility & Low & Very low & Low \\
\hline Level of side lobes & No & No & Yes \\
\hline Very fast scanning in near zone & No & Yes & 30 degrees \\
\hline Multi Beam elevation limits & No & YES & No \\
\hline Multi beam error direction & Long & Long & Short \\
\hline Observation time in near zone & Good & Good & Good \\
\hline Resistance for failure of elements & Good & $\begin{array}{c}\text { Very } \\
\text { good }\end{array}$ & Normal \\
\hline Transportability & No & Yes & No \\
\hline Split mod installation & High & Low & Low \\
\hline Maximum elevation in near zone & Normal & Normal & Low \\
\hline Side lobes at uniform ampl distribution & & & \\
\hline
\end{tabular}




\begin{tabular}{|l|c|c|c|} 
Power consumption & High & Normal & Normal \\
\hline Mechanical robustness & Normal & High & Low \\
\hline Resistance to mechanical wear & Bed & Good & Good \\
\hline $\begin{array}{l}\text { Scanning time as function of installed } \\
\text { power }\end{array}$ & $\begin{array}{c}\text { Slow (with } \\
\text { one AESA) }\end{array}$ & Normal & Fast \\
\hline Scanning time as function of size & Normal & Normal & Fast \\
\hline Suitability for long range low frequency & No & Yes & Very \\
\hline Suitability for IFF & Yes & Yes & No \\
\hline $\begin{array}{l}\text { Possibility for fast changing azimuth and } \\
\text { elevation target tracking }\end{array}$ & No & Yes & Very \\
\hline
\end{tabular}

T5. Technical complexity summary.

\begin{tabular}{|l|c|c|c|}
\hline \multicolumn{1}{|c|}{ Complexity of production } & $\begin{array}{c}\text { Mechanically } \\
\text { rotated } \\
\text { planar AESA }\end{array}$ & $\begin{array}{c}\text { Prismatic } \\
\text { system } \\
\text { of } 4 \\
\text { planar } \\
\text { AESA }\end{array}$ & $\begin{array}{c}\text { Cylindrical } \\
\text { AESA }\end{array}$ \\
\hline Complexity of mechanical construction & Normal & Normal & Hight \\
\hline Complexity of TR module & Low & Normal & Normal \\
\hline Number of TR, horn, attenuator, ADC & Low & High & Hight \\
\hline Number of phase shifter & Low & Normal & Normal \\
\hline RF divider network & Simple & Normal & Complex \\
\hline Additional switching network & No & No & Yes \\
\hline Digital signal network & Simple & Normal & Complex \\
\hline Complexity of multi beam ABF & Low & High & Very high \\
\hline Complexity of multi beam DBF & Normal & Normal & Hight \\
\hline Technical perspective & & Good & \\
\hline Phase and amplitude distribution & Simple & Normal & Complex \\
\hline optimization software complexity & Simple & Complex & Complex \\
\hline Software beam forming complexity & Simple & Complex & Complex \\
\hline Operative software complexity & & &
\end{tabular}

The AESA analysis by program confirms the theoretical assumptions regarding gain, lateral lobes, range. However, less expected, some beam deformations also appear, which significantly negatively affect the tactical characteristics.

Table $\mathrm{T} 4$ provides a qualitative overview to the tactical characteristics and $\mathrm{T} 5$ to the technical characteristics for all three types of AESA. Extremely bad 
characteristics are in red fields, and important advantages are in green. The tables show that each AESA has both red and green fields.

A comparative analysis of mechanically rotating AESA, cylindrical AESA and four-side prismatic AESA indicates that there is no absolute best solution. It is necessary to choose and design the optimal solution for a specific purpose, taking into account all the tactical and technical advantages and limitations. The state of art and component market state should also be taken into account.

Such a complex project requires software to calculate the characteristics of AESA for specific input data.

\section{References}

1. Indenbom M.V., Makhlin R.L. Radar station with a digital axisymmetric active phased antenna array as a promising direction for the development of radar with a circular view. Vestnik kontserna VKO "Almaz-Antey" [Bulletin of the "AlmazAntey" Concern]. 2017. No.3. P.24 32. http://journal.almaz-antey.ru. (In Russian)

2. Josefsson L., Persson P. Conformal Array Antenna Theory and Design. Chalmers University of Technology and Royal Institute of Technology, Sweden, Wiley-Interscience. 2006.

3. Sayidmarie K.H., Sultan Q.H. Synthesis of wide beam array patterns using random phase weights. Department of Communication Engineering College of Electronic Engineering-University of Mosul, Mosul, Iraq, ResearchGate Decembar 2013.

4. Keizer W.P.M.N. Low-Sidelobe Pattern Synthesis Using Iterative Fourier Techniques Coded in MATLAB. IEEE Antennas and Propagation Magazine. 2009. Vol.51. No.2.

5. Liu C., Ding Z., Liu X. A Low Complexity 2D Pattern Synthesis Algorithm for Cylindrical Array. International Journal of Antennas and Propagation. 2013. Article ID 352843. 
6. Ehyaie D. Novel Approaches to the Design of Phased Array Antennas. PhD thesis (Electrical Engineering\}. The University of Michigan. 2011.

7. Zavodný V., Mandlik M. Novel Antenna for 3D-Radar system. Department of Microwave Design Eldis Pardubice, s.r.o., Czech Republic, 2013.

8. Stasiowski M., Schaubert D. Broadband Array Antenna. Cobham Defense Electronic Systems Nurad Division 3310 Carlins Park Drive Baltimore, MD 21215, 2008.

9. Agrawal A.K., Kopp B.A., Luesse M.H., O’Haver K.W. Active Phased Array Antenna Development for Modern Shipboard Radar Systems. Johns Hopkins Appl. Technical Digest. 2001. Vol.22. No.4. P.600-613.

\section{For citation:}

Paunović D. Comparative analysis of cylindrical and planar aesa in 3d suveillance radar. Zhurnal

Radioelektroniki [Journal of Radio Electronics]. 2021. No.3. https://doi.org/10.30898/16841719.2021.3.8. 